Review

\title{
The Role of Autophagy and Mitophagy in Bone Metabolic Disorders
}

\author{
Shuai Wang1,3\#, Zhantao Deng1\#, Yuanchen Ma1\#, Jiewen Jin², Fangiie Qi1,3, Shuxian Li1,3, Chang Liu³, \\ Feng-Juan Lyu ${ }^{3 凶}$ and Qiujian Zheng ${ }^{\circledR}$ \\ 1. Department of Orthopedics, Guangdong Provincial People's Hospital, Guangdong Academy of Medical Sciences, 510080, China. \\ 2. Department of Endocrinology, The First Affiliated Hospital of Sun Yat-sen University. \\ 3. South China University of Technology-The University of Western Australia Joint Center for Regenerative Medicine Research, School of Medicine, South \\ China University of Technology, Guangzhou, 510006, China. \\ \#These authors contributed equally to this work.
}

$\square$ Corresponding authors: Dr. Qiujian Zheng, Department of Orthopedics, Guangdong Provincial People's Hospital, Guangdong Academy of Medical Sciences, School of Medicine, South China University of Technology, No.106, Zhongshan Second Road, Yuexiu District, Guangzhou, 510080, China, E-mail: zhengqiujian@gdph.org.cn; Dr. Feng-Juan Lyu, School of Medicine, South China University of Technology, Guangzhou, 510006, China, E-mail: Lufj0@scut.edu.cn.

(c) The author(s). This is an open access article distributed under the terms of the Creative Commons Attribution License (https://creativecommons.org/licenses/by/4.0/). See http://ivyspring.com/terms for full terms and conditions.

Received: 2020.04.03; Accepted: 2020.07.12; Published: 2020.07.30

\begin{abstract}
Bone metabolic disorders include osteolysis, osteoporosis, osteoarthritis and rheumatoid arthritis. Osteoblasts and osteoclasts are two major types of cells in bone constituting homeostasis. The imbalance between bone formation by osteoblasts and bone resorption by osteoclasts has been shown to have a direct contribution to the onset of these diseases. Recent evidence indicates that autophagy and mitophagy, the selective autophagy of mitochondria, may play a vital role in regulating the proliferation, differentiation and function of osteoblasts and osteoclasts. Several signaling pathways, including PINK1/Parkin, SIRT1, MAPK8/FOXO3, Beclin-1/BECN1, p62/SQSTM1, and mTOR pathways, have been implied in the regulation of autophagy and mitophagy in these cells. Here we review the current progress about the regulation of autophagy and mitophagy in osteoblasts and osteoclasts in these bone metabolic disorders, as well as the molecular signaling activated or deactivated during this process. Together, we hope to draw attention to the role of autophagy and mitophagy in bone metabolic disorders, and their potential as a new target for the treatment of bone metabolic diseases and the requirements of further mechanism studies.
\end{abstract}

Key words: autophagy; mitophagy; bone metabolic disorder; osteoblast; osteoclast

\section{Introduction}

Bone metabolic disorders mainly include osteolysis, osteoporosis, osteoarthritis (OA) and rheumatic arthritis (RA). Osteolysis is induced by imbalance between bone formation and bone resorption. Osteoporosis is a classic age-related bone disease characterized by low bone mineral density, deterioration of bone microstructure, and subsequent increase in bone fragility [1]. Fragility fracture is the most serious complication of osteoporosis. OA is a degenerative disease of the synovial joints, which is characterized by synovium inflammation, progressive degradation of cartilage and the associated extracellular matrix, osteophyte formation and subchondral bone sclerosis [2]. OA is the most common reason of synovial joint pain and dysfunction in the older adults [3]. The disease progression of OA affects not only articular cartilage, but also the entire joint, including subchondral bone, articular capsule, synovial membrane and surrounding muscles. The risk factors of primary $\mathrm{OA}$ include gender, ageing and obesity [4]. RA is a chronic inflammatory autoimmune disease. The pathological characteristics of RA include synovial hyperplasia, cartilage degradation, fibroblast-like synoviocytes infiltration into cartilage and bone surfaces, and subchondral bone erosion [5].

Total hip arthroplasty (THA) is a standard treatment of patients suffering from severe end-stage joint diseases such as OA, RA and severe trauma. 
THA significantly reduces pain, improves joint function and overall quality of life [6]. One late complication of THA limiting the survival of the patients is aseptic osteolysis [7], which is the major cause of THA failure. Osteolysis is caused by the imbalance between bone formation by osteoblasts and bone resorption by osteoclasts. In fact, evidences suggest that various cell types including osteoblasts, osteoclasts, macrophages, fibroblasts and lymphocytes are involved in the pathogenesis of bone metabolic disorders [8]. Immune cells, such as Th17 cells, B cells, macrophages, neutrophils, mast cells and fibroblast-like synoviocytes, are important for inducing and maintaining synovial inflammation in RA $[9,10]$. However, the underlying mechanism is not fully understood.

Autophagy is a highly conserved selfdegradative and energy dynamic recycling process in the proliferation, differentiation and maturation of eukaryotic cells, which could provide energy and basic substances for cellular homeostasis and survival through degradation of cytoplasmic misfolded or aggregated proteins, damaged organelles or macromolecules [11, 12]. In mammalian cells, there are three primary types of autophagy: microautophagy, macroautophagy and chaperone-mediated autophagy, which all eventually end in the delivery of cytoplasmic materials to the lysosome for degradation and recycling [13]. Although autophagy was originally believed to be a strictly nonselective process which randomly engulfs cytoplasmic contents, it has now been clear that autophagy also acts in a selective process [14]. Mitophagy, the mitochondrial selective autophagy, targets damaged mitochondria for degradation through receptor mediated mechanisms. Mitophagy is a normal physiological activity taking place under healthy conditions, whereas it can also be accelerated under pathological conditions [15], or specific physiological conditions to promote glycolysis in retinal development [16]. Mitophagy is required for the differentiation of reticulocyte to mature erythrocytes in rats [17], and for $\mathrm{T}$ cell development in mice thymus to remove excess mitochondria [18]. Recently, emerging evidence suggested that abnormal autophagy and mitophagy levels will break the balance of bone metabolism and play a key role in bone metabolism disorders $[19,20]$.

In this review, we expect to summarize the current knowledge on the role of autophagy and mitophagy in the bone metabolic disorders, aiming to explore whether there is a potential relationship between autophagy/mitophagy and bone metabolic disorders, which will pave the way for further studies in the future.
Osteoblasts and osteoclasts are two major types of cells contributing to bone metabolism

Bone is a metabolically active tissue with network structure constituted by various types of cells and regulated by various factors. The maintenance of a stable bone metabolism requires the continuous differentiation and maturation of different kinds of stem cells, the mineralization of osteoblasts, the phagocytosis of osteoclasts and the secretion of a series of factors to regulate the interaction between cells. These require close coordination of intracellular organelles and regulatory factors, while consuming mass biological energy. Bone metabolic homeostasis is mainly maintained by a balance between bone formation by osteoblasts and bone resorption by osteoclasts, as illustrated in Figure 1. Different types of cells in the bone serve specific skeletal functions. Chondrocytes and osteoblasts, derived from bone marrow mesenchymal stem cells, construct and shape the skeleton to get the maximal adaptability. In contrast, the osteoclasts, which are derived from hematopoietic stem cells, can maintain mineral homeostasis by resorbing cancellous bone with a large surface area. Together, they play vital roles in the development and maintenance of bone size, shape and integrity [21].

There is a complicated inter-relationship between osteoblasts and osteoclasts. The activation and maturation of osteoclast precursors can be mediated by receptor activator of nuclear factor- $\mathrm{kB}$ ligand (RANKL) and intercellular adhesion molecule1 , which are expressed on the human cellular membrane of osteoblasts and bind with RANK and lymphocyte function-associated antigen 1 on the human cellular membrane of osteoclasts, respectively [22]. Meanwhile, Tanaka et al. [22] proposed that intercellular adhesion molecule-1/lymphocyte function-associated antigen 1 adhesive pathway is a prerequisite for efficient interaction of RANKL with its receptor RANK. Moreover, Deng et al. [23] found that in the particle-induced osteolysis, wear particles could induce inflammatory responses in osteoblasts, with increased inflammatory cytokines at tissue level, including interleukin (IL)-6, IL-1 $\beta$ and tumor necrosis factor- $\alpha$ (TNF- $\alpha)[24]$, leading to significantly increased number of activated osteoclasts and consequent local osteolysis. Meanwhile, osteoclasts can in turn affect the function of osteoblasts, which is directly regulated by the intercellular interaction or indirectly regulated by the expression of multiple cytokines such as semaphorin 4D [25]. 


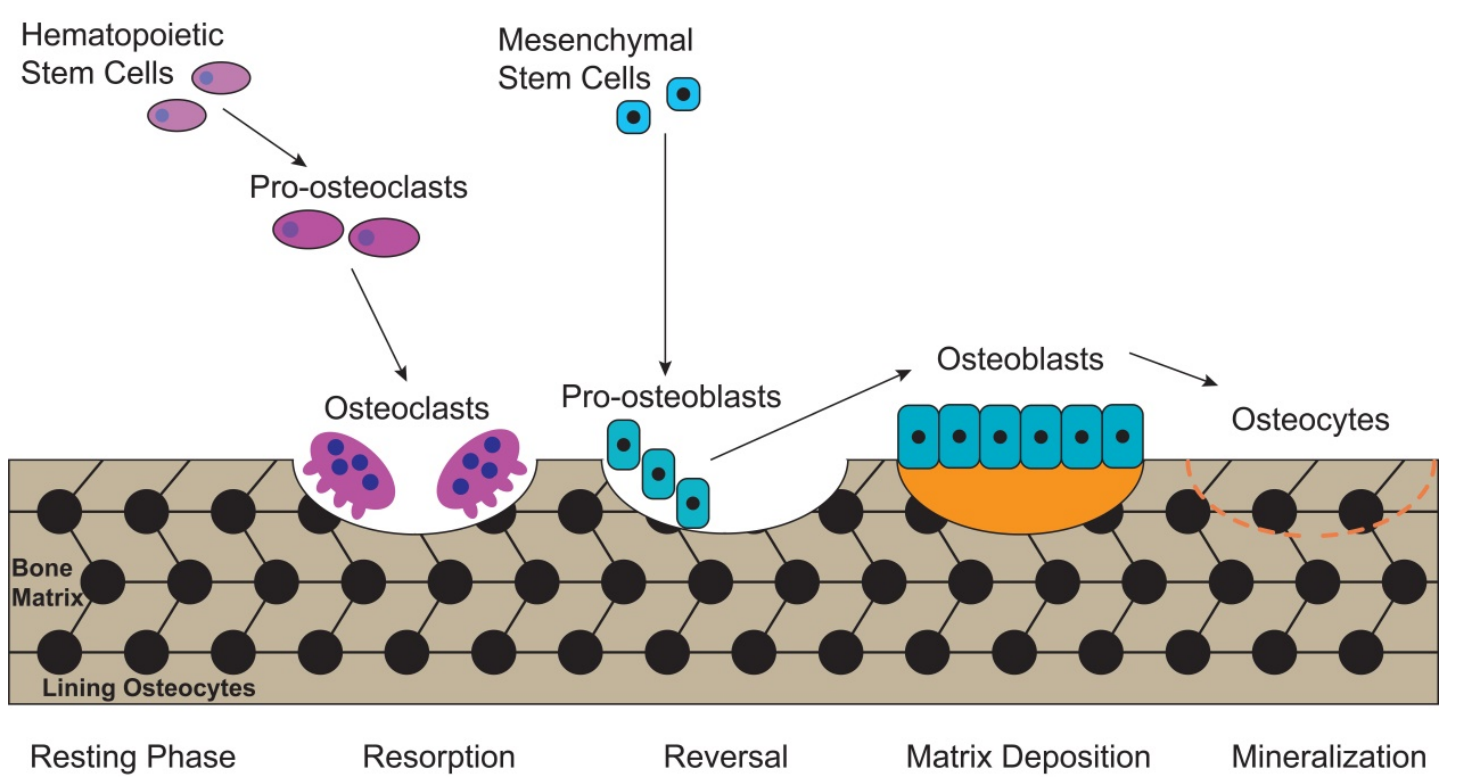

Figure 1. The process of bone metabolic homeostasis. Bone metabolic homeostasis is a continual process, including resorption, reversal, matrix deposition and mineralization. The osteoblasts which are derived from bone marrow mesenchymal stem cells, construct and shape the skeleton to get the maximal adaptability and terminally differentiate into osteocytes and form lining osteocytes. The osteoclasts, derived from hematopoietic stem cells, are multinucleated cells which can efficiently resorb bone.

\section{The definition of autophagy and mitophagy}

The term "autophagy" is derived from the Greek meaning for "eating of self". It was first presented by Deter et al. [26] over 50 years ago when they observed mitochondria and other intracellular structures in lysosome of rat liver cells treated by glucagon, a pancreatic hormone. Nowadays, we have more understanding of the process of autophagy at the molecular, cellular and tissue level [11, 12, 27, 28]. A variety of autophagy related genes (Atg) have been revealed in yeast, plants, worms, flies and mammals, demonstrating that autophagy is a fundamental function in biological evolution. ATG proteins, produced by the transcription and translation of Atg, aggregate to form specific functional complexes which are activated and recruited to membranes to initiate autophagy. Autophagy begins with the formation of the 'autophagosome', an isolated double-membraned intermediate organelle (also termed phagophore), contained by some cytoplasmic materials such as soluble materials and organelles. Autophagosome matures through fusion with the lysosome, then become autolysosome and gains the ability to degrade the contents by lysosomal acid proteases [12].

Autophagy can be classified into non-selective autophagy and selective autophagy, with the former being a response to starvation, while the latter degrading damaged proteins and organelles selectively [29]. 'Mitophagy', first proposed by Lemasters [30] in 2005, is the selective autophagy of mitochondria. Mitochondrion has cigar-shaped structure including a smooth outer mitochondrial membrane (OMM), a folded inner mitochondrial membrane (IMM), an intermembrane space and a central matrix (Figure 2) [31]. The IMM accommodates the important components of the electron transport chain (ETC) and oxidative phosphorylation, which are responsible for generating adenosine triphosphate (ATP) and reactive oxygen species (ROS) [32]. The mitochondrial ETC includes complexes I-IV, the electron transport ubiquinone and cytochrome c, meanwhile, the ETC produces the electrochemical potential energy contained in the proton $(\mathrm{H}+)$ gradient inside and outside of the IMM which can help to generate ATP from ADP and Pi by ATP synthase (complex V), as well as generate the mitochondrial membrane potential $(\Delta \Psi \mathrm{m})$ (Figure 2) $[33,34]$. Both the $\Delta \Psi \mathrm{m}$ and ROS were increased when isolated mitochondria from rat heart was treated with $\mathrm{H}_{2} \mathrm{O}_{2}$ [35], while decreased $\Delta \Psi \mathrm{m}$ usually lead to inhibit ROS production in mammalian cells [36]. Furthermore, mitochondria play a crucial role in regulating intracellular calcium transport and ion levels by providing ATP for calcium transporting proteins and calcium signaling [37]. Thus, the quantity and integrity of mitochondrial are important to maintain physiological function of cells. Mitochondrial dysfunction is represented by increasing ROS, depolarized $\Delta \Psi \mathrm{m}$, and increasing mitophagy. It is involved in various bone metabolic disorders, such as osteoporosis and OA [38-40]. Therefore, restoring mitochondrial function by reducing the ROS content in mitochondria may be a potential therapeutic target for OA. 


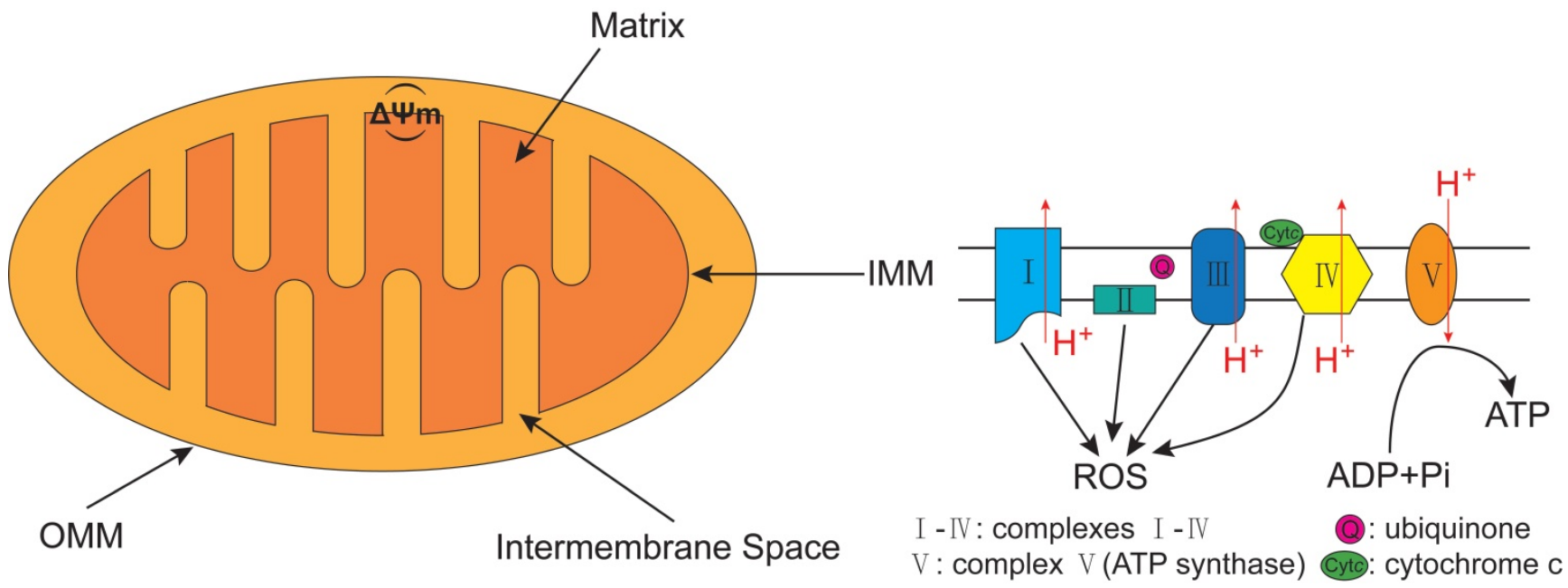

Figure 2. The structure of mitochondrion and the electron transport chain (ETC). The structure of mitochondrion includes a smooth outer mitochondrial membrane (OMM), a folded inner mitochondrial membrane (IMM), an intermembrane space and a central matrix. The ETC, including complexes I-IV, the electron transport ubiquinone and cytochrome c, is responsible for generating ATP and reactive oxygen species (ROS). The ETC produces the electrochemical potential energy contained in the proton $\left(\mathrm{H}^{+}\right)$gradient inside and outside of the IMM which can help to generate ATP from ADP and Pi by ATP synthase (complex V), as well as generate the mitochondrial membrane potential $\left(\Delta \Psi_{\mathrm{m}}\right)$.

Mitophagy contributes to tissue homeostasis via reducing intracellular ROS produced by damaged mitochondria, recycling energy by limiting the energy requirements of invalid organelles, and producing ATP during the degradation under physiological and pathological conditions [41]. In middle cortical artery occlusion-treated cerebral ischemia mice, acidic postconditioning-induced mitophagy mediated by Parkinson disease (autosomal recessive, juvenile) 2 (Parkin) can reduce neuronal injury caused by reperfusion following cerebral ischemia [42]. However, the disordered mitophagy breaks mitochondrial homeostasis and impairs cellular energy metabolism and physiological function [41]. In mouse cardiomyocytes, Overlapping with the m-AAA protease 1 homolog induced imbalance of mitochondrial fusion and fission events caused mitochondrial fragmentation mediated by optic atrophy protein 1, which facilitated removal of damaged mitochondria by mitophagy, and eventually triggered dilated cardiomyopathy and heart failure [43].

\section{The involvement of autophagy/ mitophagy in osteoblasts and osteoclasts}

Mitochondria play a vital role in the differentiation of bone precursor cells. During osteogenic differentiation of osteoblasts, a significantly increase in mitochondrial biogenesis, mitochondrial function (especially complex I activity in the mitochondrial ECT) and ATP content were found [44]. Mature osteoclasts exhibited increased mitochondrial protein content and lower intracellular ATP levels than those in osteoblasts and bone marrow macrophages [1], while the depletion of intracellular
ATP resulted in distorted mitochondrial cristae in osteoclasts and stronger phagocytosis in osteoclasts, despite accelerated apoptosis of osteoclasts.

Mitophagy plays a key role in maintaining the homeostasis of healthy and damaged mitochondria in cells [45]. The damaged mitochondria release ROS and apoptosis factors, which result in cell death or apoptosis by the activation of autophagy in osteoblasts. Impaired mitochondria can be degraded by mitophagy, which protect osteoblasts from apoptosis [46]. Sun et al. found $17 \beta$-estradiol induced mitophagy in murine MC3T3-E1 osteoblast cell line and increased cell proliferation in vitro, highlighting the significance of estrogen for the clinical treatment of osteoporosis [47]. In type 2 diabetes, loss of bone mass is a well-known risk factor correlated with osteoporotic fractures. Zhao et al. [48] found in type 2 diabetes related osteoporosis, osteoblasts had downregulated non-imprinted in Prader-Willi/Angelman syndrome region protein 2 (NIPA2), intracellular $\mathrm{Mg}^{2+}$ and activation of mitophagy, suggesting mitophagy negatively regulates osteogenesis. Yang et al. [49] used dexamethasone to treat rats with induced osteoporosis, and found that resveratrol further promoted SIRT1 (Sirtuin1)-induced mitophagy activation by suppressing phosphatidylinositol 3kinase (PI3K)/protein kinase B (AKT)/mammalian target of rapamycin (mTOR) pathway. In addition, in inflammation-associated bone loss, such as bone resorption in apical periodontitis, simvastatin inhibited mitophagy and apoptosis of regional osteoblasts to modulate the balance between tissue destruction and regeneration [50].

Autophagy plays an important role in maintaining cellular function and homeostasis by 
recycling intracellular components. Piemontese et al. [51] deleted Atg7 by an Osterix1-Cre transgene in mice to inhibit the autophagy level in osteoblasts, and found that inhibited autophagy leaded to the accumulation of endoplasmic reticulum and mitochondria and resulted in low bone mass and more fractures than the normal mice, it might be associated with decreased number of osteoblasts in the deleted Atg7 mice. In type 2 diabetes, acceleration of autophagy in osteoblasts removed the increased fragmentation of mitochondria and swollen mitochondria to protect its ability of survive and differentiation, indicating that autophagy is important to maintain the survival and function of osteoblasts to limit increased ROS and protein oxidation caused by the high glucose environment [52].

\section{Signaling pathways involved in autophagy/mitophagy in osteoblasts and osteoclasts}

Several signaling pathways have been demonstrated to involve in the regulation of autophagy/mitophagy in osteoblasts and osteoclasts. These signaling pathways as summarized in Table 1.

\section{The osteoblast and the pathway of the mitophagy: PINK1/Parkin}

The best known pathway of mitophagy is studied in mammalian cells and is mediated by PTEN-induced putative kinase 1 (PINK1) and Parkin [45]. PINK1, a serine/threonine (Ser/Thr) kinase, is required in the Parkin mediated mitophagy $[53,54]$. PINK1 possesses an N-terminal mitochondrial targeting sequence (MTS), followed by an a-helical transmembrane (TM) segment, and a Ser/Thr kinase domain (Figure 3A). Parkin, an E3-ubiquitin ligase, is selectively recruited to dysfunctional mitochondria with low membrane potential by PINK1 [55]. The PINK1 and the E3-ubiquitin ligase Parkin are encoded by PARK6 and PARK2 genes, respectively [56]. Parkin contains an N-terminal ubiquitin-like (UBL) domain, followed by three really interesting new gene (RING) domains, RING0, RING1, and RING2, and an in-between RING domain (IBR) between RING1 and RING2 (Figure 3B) [57]. Although PINK1 is expressed in most types of tissue cells, the expression level is usually imperceptible under healthy conditions.

In healthy circumstances (Figure 4A), while PINK1 is recruited to the healthy mitochondria, the $\mathrm{N}$-terminal MTS of PINK1 is translocated across the OMM and IMM through the translocase of the outer membrane (TOM) and expose to central matrix with the aid of $\Delta \Psi \mathrm{m}$ [57], and MTS is cleaved by mitochondrial processing peptidase (MPP) in the mitochondrial matrix, while the TM segment of PINK1 is cleaved by presenilin-associated rhomboidlike protease (PARL) in the inner membranes [58]. This leaves an instable amino acid at the $\mathrm{N}$-terminal of PINK1 which is then separated from the mitochondria to the cytosol for recognition by $\mathrm{N}$-end rule pathway and rapid degradation by the ubiquitin protease system [59]. Meanwhile, the UBL domain of Parkin negatively autoregulates its own E3-ubiquitin ligase activity at the N-terminus [60] to keep Parkin in an inactive state in the cytoplasm. Therefore, mitophagy is inhibited in normal cells with mitochondrial respiratory function.

\section{(A) PINK1}

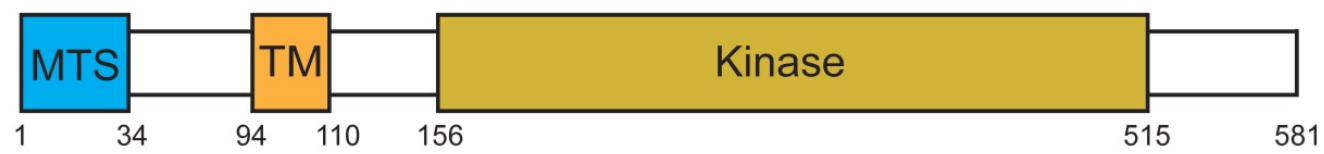

(B) Parkin

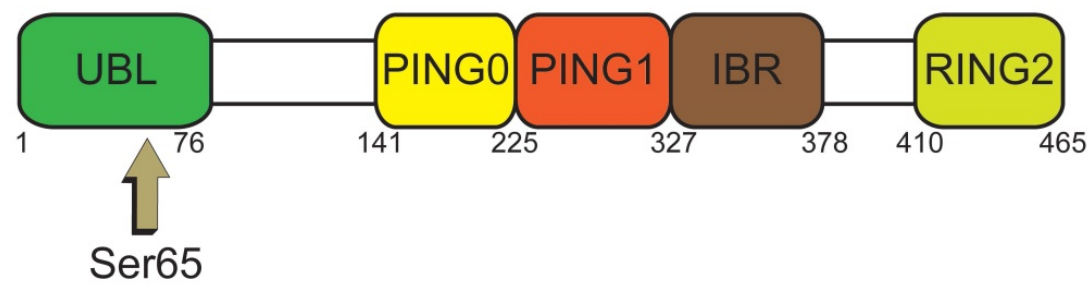

Figure 3. The structure domain of PTEN-induced putative kinase 1 (PINK1) and Parkin. (A) PINK1 possesses an N-terminal mitochondrial targeting sequence (MTS), an a-helical transmembrane (TM) segment, and a Ser/Thr kinase domain (Kinase). (B) Parkin contains an N-terminal ubiquitin-like (UBL) domain, three really interesting new gene (RING) domains (RING0, RING1, and RING2), and an in-between RING (IBR) domain between RING1 and RING2. PINK1 directly activates Parkin by phosphorylated Serine 65 (Ser65) in the UBL domain of Parkin. 
Table 1. Major autophagy/mitophagy regulation signaling pathways in the osteoblasts, MSCs and osteoclasts

\begin{tabular}{ll}
\hline Cells & \multicolumn{2}{l}{ Mechanisms } \\
\hline Osteoblasts MSCs & \\
PINK1/Parkin & 1. PINK1 escapes from degradation and instead steadily binds with TOM, which recruits Parkin and Parkin ubiquitinates OMM proteins \\
& (TOM20, mitofusins, VDAC1), recognized by autophagy receptors, such as p62/SQSTM1 and LC3, then initiate mitophagy; \\
& 2. PINK1/Parkin regulates the osteogenic differentiation of MSCs by PINK1/Parkin-induced mitophagy; \\
& 3. In hypoxia-induced apoptosis of apical periodontitis, increased PINK1/Parkin was found which can up-regulate mitophagy activity. \\
SIRT1 & 2. SIRT1 directly participates in the regulation of metabolism and biogenesis of mitochondria; \\
& 2. SIRT1 reduces the acetylation level of FOXO3a then increase the level of FOXO3a and SOD2 to achieve enhanced osteogenesis and reduced \\
& senescence of MSCs; \\
& 3. SIRT1 dramatically reduces the expression of particle-induced inflammatory cytokines and the apoptosis of osteoblasts via NF-kB and p53 \\
signaling. &
\end{tabular}

Osteoclasts

Beclin-1/BENC1 1. Wear particles induce CD147 expression which can in turn induce Beclin-1 mediated autophagy to promote osteoclast formation;

2. GIT1 promotes Bcelin-1 phosphorylation at Thr119 to disrupt the binding of Beclin-1 and Bcl2, and induce autophagy;

3. KLF2 regulates autophagy by reducing BECN1 expression via decreased histone H3K9 and H4K8 acetylation in the promoter region of Becn1 during osteoclastogenesis.

p62/SQSTM1 1. p62/SQSTM1 negatively correlates with LC3 accumulation and F-actin ring formation for regulating autophagic activation;

2. p62/SQSTM1 is a key regulator of ubiquitinated protein turnover by affecting NF-kB signaling, as well as positively stimulating NF-kB signaling and the oxidative stress-induced Keap1/Nrf2 pathway;

mTOR 1. OPG enhances p-AMPKa and TSC2 expression. The activation of TSC2 by AMPK reduces mTOR activity via inhibiting Rheb activity, hence inhibits the mTOR mediated phosphorylation of p70S6K and reduces its inhibition on autophagy;

2. Suppression of AMPK/mTOR/ULK1 signaling axis negatively regulates autophagy in diabetes;

3. Activation of PI3K/AKT/mTOR pathway inhibits autophagy in hydrogen sulfide treated osteoclasts.

HIF-1a 1. Activation of HIF-1a-dependent BNIP3 promotes hypoxic-induced activation of autophagy;

2. The regulatory axis of HIF-1a-miRNA-20a-Atg16l1 activated autophagy in hypoxia induced osteoclast differentiation.

The process of autophagy/mitophagy is vital to proliferation and function of osteoblast, MSCs and osteoclast. The dysregulation of autophagy/mitophagy can lead to damaged mitochondria and accumulation of abnormal autophagy regulation factor, and induce apoptosis of osteoblasts or osteoclastogenesis in the bone metabolism disorders. The table lists the major regulated autophagy/mitophagy signaling pathways in the osteoblasts, MSCs and osteoclasts and possible potential pathogenic mechanisms.

Abbreviations: AKT: protein kinase B; AMPK: AMP-activated protein kinase; Atg: autophagy related genes; Bcl2: B-cell lymphoma-2; Beclin-1: Bcl-2 interacting coiled-coil protein; BNIP3: BCL2/adenovirus E1B 19 kDa protein-interacting protein 3; FOXO3a: Forkhead box O3a; GIT1: G-protein-coupled receptor kinase-interacting protein 1; HIF-1a: hypoxia-inducible factor-1a; JNK: Jun N-terminal kinase; Keap1: Kelch-like ECH-associated protein 1; KLF2: kruppel-like factor 2; LC3: light chain 3; MAPK8: mitogen-activated protein kinase 8; MSCs: mesenchymal stem cells; mTOR: mammalian target of rapamycin; NF-kB: nuclear factor-kB; Nrf2: NF-E2-related factor 2; OMM: outer mitochondrial membrane; OPG: Osteoprotegerin; p62/SQSTM1: Sequestosome 1; p70S6K: 70-kDa ribosomal protein S6 kinase; Parkin: Parkinson disease (autosomal recessive, juvenile) 2; PI3K: phosphatidylinositol 3-kinase; PINK1: PTEN-induced putative kinase 1; Rheb: Ras homolog enriched in brain; ROS: reactive oxygen species; Ser: Serine; SIRT1: Sirtuin 1; SOD2: superoxide dismutase 2; TOM: translocase of the outer membrane; TSC2: tuberous sclerosis complex 2; ULK1: UNC-51 like autophagy activating kinase 1; VDAC1: voltage-dependent anion channel 1.

Whereas, when the mitochondria are damaged by excessive ROS (Figure $4 \mathrm{~B}), \Delta \Psi \mathrm{m}$ is depolarized which results in the escape of MTS/TM of PINK1 from MPP/PARL-induced processing and the rest of PINK1 escape of $\mathrm{N}$-end rule pathway-dependent degradation. Instead, PINK1 steadily binds with the TOM [61], providing a platform for homodimer formation which is subsequent auto-phosphorylated. The resulting complex has a high kinase activity that promotes Parkin recruitment onto the damaged mitochondria [62]. When PINK1 directly convert Parkin to an active state (formation of a ubiquitinthioester intermediate) by phosphorylated Ser 65 in the UBL domain of Parkin (Figure 3B) [63], this posttranslational modification eliminates the autoinhibition of the UBL domain and induces Parkin activation. Activated Parkin ubiquitinates many OMM proteins, such as TOM20, mitofusins and voltage-dependent anion channel 1 [64-66], for recruitment to phagophores that mature in to autophagosomes then fuse with lysosomes resulting in degradation of damaged mitochondria [67]. Sufficient evidence indicates that ubiquitinated OMM proteins can bind to the phagophore by either direct binding to the autophagic adaptor protein light chain 3-II (LC3-II), which is embedded in the phagophore membrane [68], or through p62/Sequestosome 1 (SQSTM1), which contains a LC3 interacting domain to bind to LC3, then promote mitophagy [69]. PINK1 can either regulate Parkin activation by another important substrate, ubiquitin [15, 57, 70]. PINK1 mediates the phosphorylation at Ser65 of ubiquitin, then the phospho-ubiquitin Ser65 promotes activation of Parkin phosphorylated at Ser65 by PINK1, furthermore phosphorylation of both Parkin Ser65 and ubiquitin Ser65 greatly promote ubiquitin binds to Parkin to maximally activate E3-ubiquitin ligase activity of Parkin, thus ubiquitin is associated with efficient translocation of Parkin to damaged mitochondria (Figure 4B) [70-73]. Evidence suggests that in addition to p62/SQSTM1, the E3-ubiquitin ligase Parkin stimulated ubiquitination of various OMM proteins [15] and mediated mitochondria as the cargo sequestrated into the autophagosomes by the Bcl-2/ adenovirus E1B $19 \mathrm{kDa}$ interacting protein 3 (BNIP3) binding with the LC3 and selective damaged mito- 
chondria [74]. The formed mitochondrial autophagosome will fuse with the lysosome to form the mitochondrial autolysosome resulting in degradation of the damaged mitochondria, thereby achieves quality control of mitochondria.

PINK1 and Parkin are the most extensively studied mitophagy-associated proteins. In the familial Parkinson's disease, loss-of-function mutations in Parkin and PINK1 caused accumulation of dysfunctional mitochondria and leaded to nigral neurodegeneration and early-onset Parkinson's disease, which indicates PINK1 and Parkin as key mediators of mitochondrial homeostasis and mitophagy $[75,76]$. The down-regulated expression of Parkin led to deteriorated osteogenic differentiation with dramatically reduced expression of bone morphogenetic protein 2 and collagen I in adiposederived mesenchymal stem cells (MSCs) [77].

\section{The osteoblast and the pathway of the mitophagy: SIRT1}

The Sirtuins are a highly conservative protein family of nicotinamide adenine dinucleotide (NAD) dependent enzymes of deacetylases. They regulate the longevity of lower organisms, and regulate cellular and metabolic functions including stress resistance, genomic stability, tumorigenesis and energy metabolism, in mammals [78]. Sirtuin 1 (SIRT1) was originally isolated in a screen for silencing factors and is the mammalian homolog of yeast silent information regulator 2 [79]. SIRT1 is an important regulatory factor of bone metabolism. Cohen-Kfir et al. [80] found that Sirt1 knockout in female mice resulted in significant bone loss and decreased osteogenesis of MSCs and increased marrow adipogenesis. Sun et al. [81] attested that SIRT1 overexpression in MSCs repressed the acetylation of Forkhead box O3a (FOXO3a) and increased the expression of FOXO3a and superoxide dismutase 2 (SOD2), which promoted osteogenesis and reduced senility of MSCs. Meanwhile, wear particles could reduce the expression of SIRT1 in osteoblasts and osteolysis animal models [23]. In contrast, the enhanced expression of SIRT1 in osteoblasts can dramatically reduce the expression level of particle-induced inflammatory cytokines and apoptosis of osteoblasts by NF-кB (nuclear factor-kB) and p53 signaling [23].

(A) Healthy mitochondria

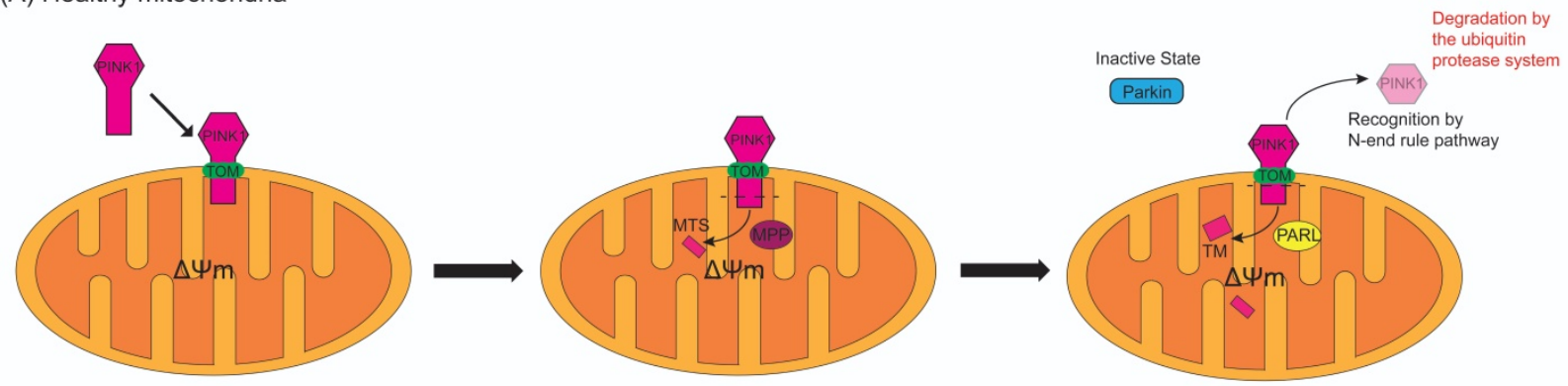

(B) Damaged mitochondria
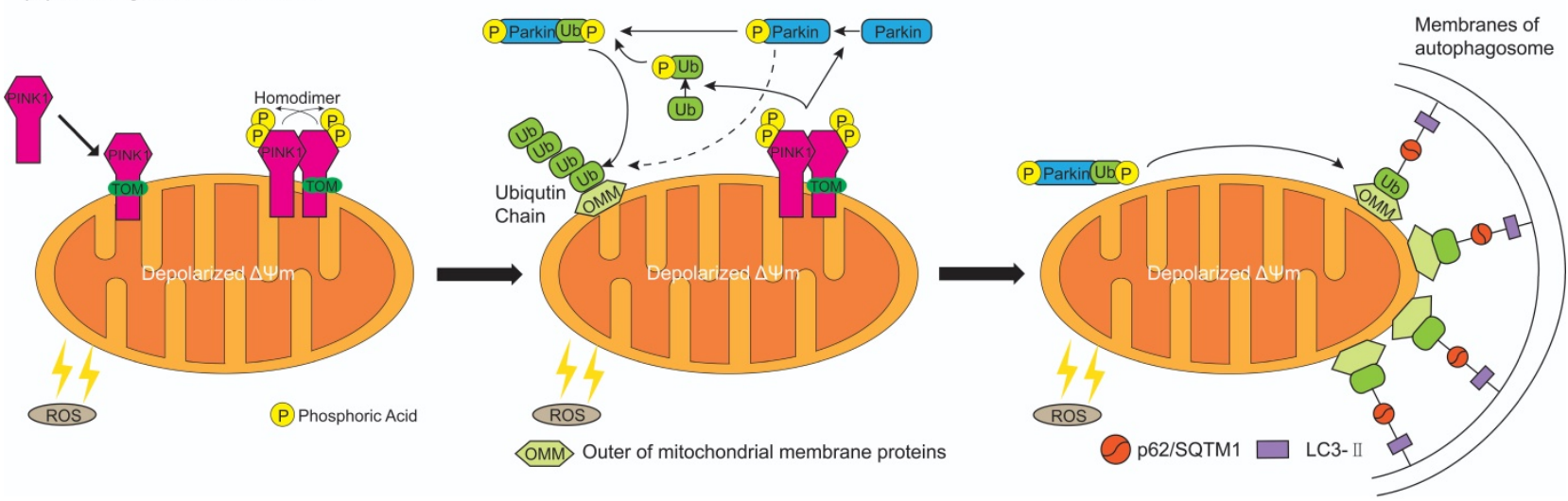

Figure 4. The process of PINKI and Parkin mediated mitophagy on healthy and damaged mitochondria. (A) While PINKI is recruited to the healthy mitochondria, N-terminal mitochondrial targeting sequence (MTS) of PINK1 is translocated across the mitochondrial membranes through the translocase of the outer membrane (TOM), and is exposed to central matrix depending on $\Delta \Psi_{\mathrm{m}}$. Then MTS is cleaved by mitochondrial processing peptidase (MPP) in the mitochondrial matrix and the TM segment of PINK1 is cleaved by presenilin-associated rhomboid-like protease (PARL) in the inner membranes. The rest of PINK1 with an instable amino acid at the $\mathrm{N}$-terminal is released to cytosol, recognized by $\mathrm{N}$-end rule pathway and rapid degraded by the ubiquitin protease system. As a result, Parkin keep in an inactive state in the cytosol. (B) When the mitochondria are damaged by the reactive oxygen species (ROS), $\Delta \Psi_{\mathrm{m}}$ is depolarized and MTS cannot reach into the matrix which results in the escape of PINK1 from MPP/PARL-induced processing and N-end rule pathway-dependent degradation, therefore PINK1 steadily binds with the TOM. Two molecules of PINK1 form a homodimer and are intermolecularly phosphorylated to become highly active. Then PINK1 induces the phosphorylation of Parkin and ubiquitin at Ser65. Activated Parkin combine with or without phosphorylated ubiquitin can ubiquitinate many OMM proteins, but phospho-ubiquitin binded to Parkin can maximally activate E3-ubiquitin ligase activity of Parkin. In consequence ubiquitinated OMM proteins bind to the autophagosome by either direct binding to the LC3-II embedded in the membrane of autophagosome, or indirectly through p62/ Sequestosome 1 (SQSTM1), which contains a LC3 interacting domain and can bind to LC3, then promotes mitophagy. 
SIRT1 regulates the quality of mitochondria through mitophagy via different pathways in different cells. Deacetylation of the peroxisome proliferator-activated receptor gamma coactivator-1a (PGC-1a) mediated by SIRT1 plays a key role in mitochondrial metabolic control and mitochondrial biogenesis [82]. However, SIRT1 is also reported to associate with mitochondrial degradation through mitophagy [83]. Nicotinamide can enhance mitophagy and the effect is achieved through increasing the $\mathrm{NAD}^{+} / \mathrm{NADH}$ ratio and activating SIRT1 [84]. SIRT1 also regulates mitophagy by PINK1/Parkin pathway. A recent study on ischemic reperfusion in rat hearts demonstrated that resveratrol sequentially activated SIRT1, SIRT3, FOXO3a, PINK1, and PINK1 activated Parkin, leading to increased mitophagy [85]. In the luminal epithelium of the human prostate cancer, loss of SIRT1 induced increased acetylation of SOD2 and reduced SOD2 activity, subsequently enhanced ROS production which induced the recruitment of Parkin to the mitochondria triggering mitophagy [86]. In contrast, SIRT1 restoration could delay Parkin translocation to the mitochondria and reduce mitophagy [86].

\section{The osteoblast and the pathway of the autophagy: MAPK8/FOXO3}

FOXO is a family of transcription factors playing a vital role in the cellular defense against oxidative stress. The FOXO family comprises four members: FOXO1, FOXO3, FOXO4 and FOXO6 [87]. GomezPuerto et al. [87] studied osteogenic differentiation of human MSCs and found that FOXO3 was activated by ROS, which was caused by increased mitochondrial metabolism for sufficient energy of differentiation, and the process was mainly dependent on mitogen-activated protein kinase 8 (MAPK8)-induced Ser294 phosphorylation of FOXO3, and FOXO3 activation is important in the control of ROS levels via the activation of autophagy. In fluoride-induced apoptosis of osteoblast, MAPK/JNK (Jun N-terminal kinase)-dependent autophagy acts as a protective role against apoptosis [88]. Endoplasmic reticulum to nucleus signaling 1 (ERN1) can trigger autophagy, Wang et al. [19] observed that CoCrMo metal particles induced autophagy was mediated by MAPK8 after activated by ERN1.

\subsubsection{The osteoclast and the pathway of the autophagy: Beclin-1/BECN 1}

Wear particles induced osteoclastogenesis is always an important cause of osteolysis in artificial joint replacement. Several molecules, including CD147, G-protein-coupled receptor kinase-interacting protein 1 (GIT1), IL-17A, kruppel-like factor 2 (KLF2), and tumor necrosis factor receptor-associated factor 6 (TRAF6) are reported to involve in the regulation of the autophagy in osteoclasts. Su et al. [89] found CD147 induced by wear particles activated autophagy, with increased level of Beclin-1 (Bcl-2 interacting coiled-coil protein) and soluble RANKL to promote osteoclastogenesis. Under starvation condition, GIT1 contributed to autophagy in osteoclasts through the disruption of the binding of Beclin1 with B-cell lymphoma-2 (Bcl2) by promoting the phosphorylation of Beclin1 at Thr119 [90]. IL-17A, an inflammatory cytokine, is also involved in RANKL-induced osteoclastogenesis by regulating the autophagic Beclin-1 activity [91]. KLF2, a member of the zinc finger transcription factor family, which critically regulates embryonic lung development, and the function of endothelial cells, B-cells, T-cells and monocytes [92], could regulate autophagy in myeloid cells, which suppresses BECN1 expression and decreases histone $\mathrm{H} 3 \mathrm{~K} 9$ and $\mathrm{H} 4 \mathrm{~K} 8$ acetylation in the promoter region of Becn 1 during osteoclastogenesis [93]. TRAF6, as an E3 ubiquitin ligase, mediated ubiquitination of Beclin1 at Lys117 for RANKLstimulated osteoclast differentiation [94]. Overall, Beclin-1/BECN1 pathway plays an important role in the autophagy of osteoclasts.

\section{The osteoclast and the pathway of the autophagy: p62/SQSTM1}

SQSTM1/p62 is a characterized adaptor protein for autophagy in RANKL-induced osteoclastogenesis. During RANKL-induced osteoclast differentiation, the expression and localization of p62/SQSTM1 negatively correlated with LC3 accumulation and F-actin ring formation for regulating autophagic activation [95]. Kaempferol, a flavonoid compound, can inhibit autophagy by degradation of p62/ SQSTM1 and activate cell apoptosis during RANKLinduced osteoclastogenesis in murine macrophage (RAW264.7) cells [96]. Paget's disease of bone is a bone disorder characterized by focal areas of abnormal, excessive bone turnover, specifically increased bone resorption and resulting in disorganized bone formation [97]. In Paget's disease of bone, p62/SQSTM1 is a key regulator of ubiquitinated protein turnover through autophagy and ubiquitin-proteasome system, as well as positively stimulating NF- $\mathrm{KB}$ signaling and the oxidative stress-induced Kelch-like ECH-associated protein 1/NF-E2-related factor 2 (Keap1/Nrf2) pathway [98]. 
(A). OPG/RANKL/RANK

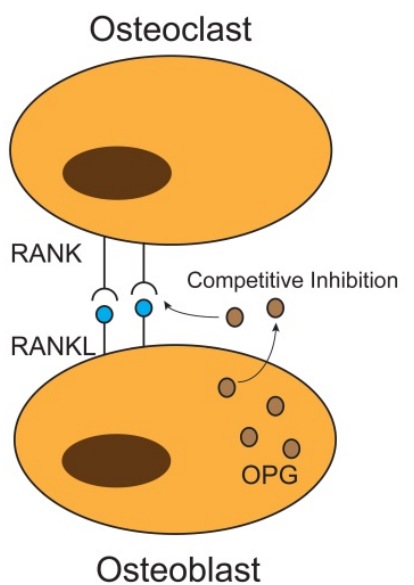

(B). AMPK/mTOR/p70S6K

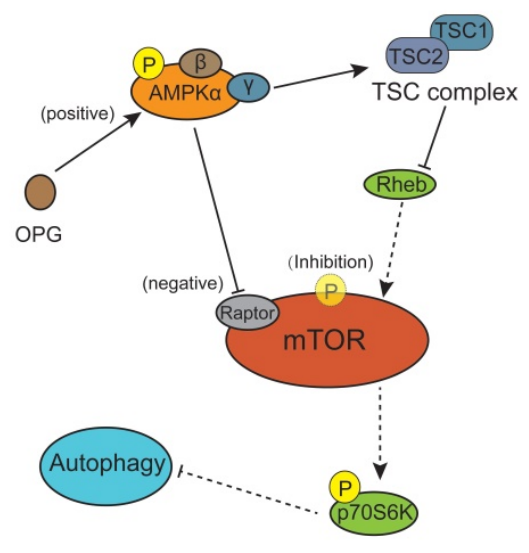

(C). AMPK/mTOR/ULK1

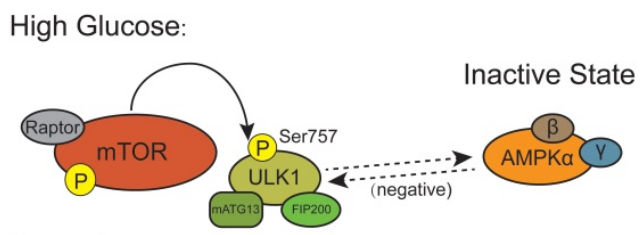

Starvation:

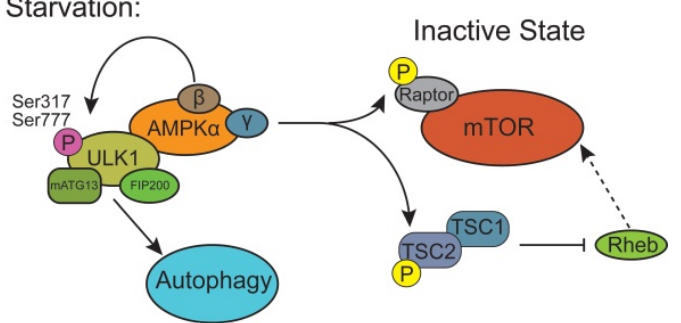

Figure 5. The models for mammalian target of rapamycin (mTOR) behaviors on autophagic signaling pathway activation during osteoclast differentiation and formation. (A) Osteoprotegerin (OPG) is a decoy receptor for the RANKL and competitively inhibit osteoclasts differentiation and maturation through blocking the interaction between RANKL and RANK. (B) OPG inhibits osteoclastogenesis and bone resorption by enhancing autophagy through activating AMPK/mTOR/p70S6K signaling pathway. OPG activates AMP-activated protein kinase (AMPK) and downstream tuberous sclerosis complex 2 (TSC2). Activated AMPK inhibits mTOR through either phosphorylating Raptor on mTOR or promoting TSC1/TSC2 complex formation to inhibit Ras homolog enriched in brain (Rheb), which can induce mTOR activation. Reduction of activated mTOR activate autophagy indirectly by nonactivated 70-kDa ribosomal protein S6 kinase (p70S6K). (C) The AMPK/mTOR/UNC-51 like autophagy activating kinase 1 (ULK1) signaling pathway mediated autophagy is involved in the regulation of energy metabolism in osteoclastogenesis. Under high glucose condition, activated mTOR phosphorylates ULK1 at Ser757, which inhibits the interaction between ULK1 and AMPK, to suppress autophagy. Under glucose starvation conditions when energy supply is exhausted, activated AMPK phosphorylates TSC2 and Raptor, which inhibits the activation of mTOR, then signal transduction from AMPK to ULK1 is restored. AMPK phosphorylates and activates ULK1 at multiple residues (Ser317 and Ser777), the activated ULK1/mATG13/FIP200 protein kinase complex lead to autophagy.

\section{The osteoclast and the pathway of the autophagy: mTOR}

Mammalian target of rapamycin (mTOR) is one of several signal sensors to control the autophagic signaling pathway activation during osteoclast differentiation and formation (Figure 5). Osteoprotegerin (OPG) is a glycoprotein secreted by osteoblasts and is a key regulatory factor of bone activity. It is a decoy receptor for the RANKL with an equivalently high affinity as RANKL for its receptor RANK and can inhibit osteoclast differentiation and bone resorption by blocking the interaction between RANKL and RANK (Figure 5A) [99-102]. Autophagy is found to participate in this process via AMPactivated protein kinase (AMPK)/mTOR/70-kDa ribosomal protein $\mathrm{S} 6$ kinase (p70S6K) signaling pathway (Figure 5B) [103]. OPG enhanced the expression of $\mathrm{p}$-AMPKa/AMPKa and downstream tuberous sclerosis complex 2 (TSC2). In the absence of energy or exogenous stimulus, AMPK can enhance TSC2 activation, promoting TSC1/TSC2 complex formation to inhibit Ras homolog enriched in brain (Rheb) activation [104]. Tong et al. [103] found the OPG-induced increased TSC2 combined with TSC1 to form TSC complex, which deactivated the small G protein Rheb, thereby reducing mTOR activity. Owing to the activation of AMPK protein could inactivate mTOR activity through inhibiting Rheb, reduction of $\mathrm{p}$-mTOR/mTOR expression activated autophagy accompanied by indirectly reducing $\mathrm{p}$ -
p70S6K/p70S6K expression [102]. In other studies, suppression of the AMPK/mTOR/UNC-51 like autophagy activating kinase 1 (ULK1) signaling axis decreases autophagy in high-glucose-treated osteoclasts [105], which is attributed to that activated mTOR phosphorylates ULK1 at Ser757 under the high-glucose condition, which inhibits the interaction between ULK1 with its upstream activating factor AMPK then autophagy in response to energy stresses is suppressed (Figure 5C) [106]. Under glucose starvation conditions when cellular energy is exhausted, AMPK is activated and phosphorylate two important mTOR factors, TSC2 and Raptor (Figure 5C) [107]. In this condition, energy-consuming mTOR signaling pathway is turned off and activity is restrained, which allows signal transduction from AMPK to ULK1. AMPK phosphorylates and activates ULK1 at multiple residues (Ser317 and Ser777), forming activated ULK1/mATG13/focal adhesion kinase family interacting protein of $200 \mathrm{kDa}$ (FIP200) protein kinase complex lead to autophagy [108-110].

\section{The osteoclast and the pathway of the autophagy: HIF-1 $\alpha$}

Under hypoxic conditions, hypoxia-inducible factor-1a (HIF-1a) acts as a crucial role in the activation of autophagy. Zhao et al. [111] found that the up-regulated expression of HIF-1a-dependent Bcl-2/adenovirus E1B $19 \mathrm{kDa}$ interacting protein 3 (BNIP3) was involved in hypoxic-induced activation of autophagy, which finally led to osteoclastogenesis. 
Under hypoxic conditions, in addition to acting as a protein signaling pathway to regulate autophagy, HIF-1a also mediated miRNAs which are involved in the regulation of autophagy in osteoclasts [112]. During hypoxia induced osteoclast differentiation, up-regulated HIF-1a suppressed the expression of miRNA-20a on transcriptional level. However, miRNA-20a directly bound at the 3'-untranslated region of Atg16l1 to negatively regulate ATG16L1 of autophagy, resulting in the regulatory axis of HIF-1a-miRNA-20a-Atg16l1 activated autophagy in hypoxia induced osteoclast differentiation [112].

\section{The involvement of autophagy/ mitophagy in bone metabolic disorders}

\section{Osteolysis}

Osteolysis is the major cause of THA failure with aseptic loosening as a severe complication. Its pathophysiology is not fully understood. The main pathogenic factor is prosthesis wear, resulting in wear particles around prosthesis and bone. In addition, other factors may contribute to the occurrence of osteolysis, such as the material and design of the prosthesis itself, the procedures performed during implantation, and a lack of connectivity at the interface between the implant and bone [113]. Wear particles could provoke some pathologic conditions, such as inflammation, formation of foreign body granulomas and bone resorption by activated osteoclast, which ultimately lead to osteolysis [113]. Current evidence suggests autophagy is an important factor in the pathogenesis of osteolysis [7]. Recent evidence indicated that autophagy can be triggered by wear particles in the three main cell types involved in osteolysis, including osteoclasts, osteoblasts, and macrophages [19, 20,24]. Autophagy could induce the secretion of proinflammatory cytokines such as TNF-a, IL-6 and IL-8 or high mobility group box 1 in macrophages and osteoblasts, which have been implicated in the pathogenesis of aseptic loosening [114]. Wear particles could stimulate the apoptosis of osteoblasts through enhancing their autophagy level, leading to osteolysis in a mouse calvarial resorption animal model [19]. Wear alloy particles also induced differentiation of osteoclasts from bone marrow monocytes through stimulating autophagy in osteocytes which could reduce the expression of interferon- $\beta$ [20]. However, Li et al. [115] found that nano-sized $\mathrm{Al} 2 \mathrm{O} 3$ particle induced autophagy in fibroblasts which reduced RANKL expression and negatively regulated the differentiation and maturation of osteoclasts, hence alleviated osteolysis. Furthermore, a few investigations of autophagy involvement in osteolysis in animal models indicated that the inhibition of autophagy decreased osteolysis severity [19, 24, 50, 51, 115]. Therefore, inducing autophagy in fibroblasts to limit the activation of RANKL on osteoclasts may be a potential therapeutic target for the prevention and treatment of osteolysis.

\section{Osteoporosis}

The primary osteoporosis includes postmenopausal osteoporosis and senile osteoporosis. $17 \beta$-estradiol deficiency is one of the main causes of postmenopausal osteoporosis, which can be treated by $17 \beta$-estradiol to protect osteoblasts by inducing mitophagy via the G protein-coupled receptor 30extracellular regulated protein kinases 1/2 (ERK1/2) signaling pathway, however the protective effect could be abolished when the cells were pretreated with G15, a selective $G$ protein-coupled receptor 30 antagonist [116]. In addition to the signaling pathways involved in the imbalance between bone formation and resorption in osteoporosis, such as Wingless/Integrated (Wnt) pathway, Bone morphogenetic protein/Mothers against decapentaplegic homolog (BMP/Smad) pathway, RANKL/RANK pathway and TNF-a pathway [117], the mTOR pathway mediated autophagy (Figure 5) also regulates the regenerative function of MSCs to control the development of postmenopausal osteoporosis [118]. Furthermore, oxidative stress, as a crucial primary factor for impaired osteoblastic bone formation in the osteoporosis, could be alleviated by early autophagy through endoplasmic reticulum stress pathway, including glucose-regulated protein 78 (GRP78) and protein kinase-like endoplasmic reticulum kinase (PERK) [119]. SIRT1 is a key regulatory factor of mitophagy and can dramatically promote the autophagy level in osteoblasts to regulate bone metabolism. The drug candidates targeting SIRT1 may have a promising effect in treating osteoporosis and other bone metabolic disorders [120].

\section{OA}

Autophagy plays an important role in the pathological development of OA. Chondrocyte apoptosis is involved in the pathogenesis of cartilage degeneration in OA. Chen et al. [121] found that conditioned medium of MSCs ameliorated the pathological development of $\mathrm{OA}$ in the rats by maintaining subchondral bone structure, producing more cartilage matrix, and inhibiting chondrocytes apoptosis with enhanced autophagy. Other evidences show that the enhancement of autophagic flux by isopsoralen could ameliorate IL-1 $\beta$-stimulated apoptosis in rat chondrocytes [122]. Furthermore, apoptosis of rat chondrocytes induced by hydrogen 
peroxide could be alleviated by global adiponectininduced autophagy through the AMPK/mTOR pathway [123]. Hydroxytyrosol (HT) has been used in the management of inflammatory diseases due to its anti-oxidative and anti-inflammatory pharmacological activities [124]. Zhi et al. [125] found that HT could promote SIRT6-mediated autophagy, and control the progression of OA caused by inflammatory factors, such as IL-1 $\beta$, IL- 6 and TNF- $\alpha$. Meanwhile, Cetrullo et al. [126] also indicated that HT prevented oxidative stress-induced apoptosis of chondrocyte by inducing autophagy in a SIRT1dependent as well as SIRT1-independent manners. More recently, transcription factor EB (TFEB) is identified as an important regulator of the autophagic flux by inducing lysosome biogenesis and promoting the autophagosome formation and fusion with lysosome [127]. Accumulating evidence suggests that TFEB overexpression could ameliorate cartilage degradation post-surgery, through restraining the apoptosis and senescence of chondrocyte, and enhancing the autophagic flux [128]. These researches suggest that autophagy plays a key role in the pathological development of OA.

The inflammatory process in OA also involves in the regulation of autophagy. As an important inflammation factor, IL-1 $\beta$ can induce experimental OA in chondrocytes [129]. Inflammation reduced the autophagy level in rat chondrocytes [130], whilst activating autophagy attenuated the inflammation and the secretion of inflammatory factors in articular OA chondrocytes. In IL-1 $\beta$ stimulated OA chondrocytes, sucrose could activate autophagy through the activation of AKT/mTOR/P70S6K signaling pathway to block IL-1 $\beta$-induced apoptosis and inflammatory reaction [131]. Ansari et al. [132] indicated the extract of Butea monosperma (Lam.) flower has a strong ability to activate autophagy via suppression of mTOR pathway and suppressing IL-1 $\beta$ induced the expression of IL-6 and matrix-metalloproteases-3, -9 and -13 in human OA chondrocytes. Ozone treatment could suppress IL- $1 \beta$ induced IL- 6 and TNF- $\alpha$ mRNA expression through promoting autophagy by activation of the AMPK/mTOR signaling pathway in OA chondrocytes [133]. In addition, miRNA-335-5p alleviates $\mathrm{OA}$ chondrocytes inflammation by activation of autophagy to reduce the gene expression of IL-1 $\beta$, IL-6 and TNF- $\alpha$ [134]. Icariin protect against OA by suppressing inflammatory cytokines (IL-1, IL-6 and IL-12) and cells apoptosis, through activation of autophagy via NF-kB inhibition [135], Butein $\left(2^{\prime}, 3,4,4^{\prime}\right.$-Tetrahydroxychalcone) activate autophagy through activating AMPK/TSC2/ULK1/mTOR pathway to inhibit IL-1 $\beta$ stimulated IL-6 expression in human chondrocytes with OA [136]. All these evidences suggest that $\mathrm{OA}$ is associated with inflammation through suppressing autophagy.

In addition to autophagy, recent researches have also tried to explore the role of mitochondrial pathology in OA [137]. Wang et al. [138] demonstrated that the prevention effect of metformin against OA was achieved through the upregulation of SIRT3-mediated PINK1/Parkin-dependent mitophagy. Huang et al. [139] found that in the monosodium iodoacetate-treated chondrocytes, which is a commonly used model for mimicking OA progression, zinc could reverse the negative effects of monosodium iodoacetate on energy metabolism of chondrocytes and eventually upregulate the PINK1-dependent selective mitophagy. In contrast, Shin et al. [140] indicated that in the chondrocytes derived from OA rat model and human primary OA patients, increased PINK1-mediated mitophagy contributed to degeneration of OA cartilage. Li et al. [141] indicated that plant homeodomain finger protein 23 (PHF23), a new autophagy inhibitor by promoting the degradation of E3 ligase, was overexpressed in human OA cartilage and synovium. Another study indicated that knockout of PHF23 prevent the chondrocytes apoptosis against IL-1 $\beta$ induced OA by increasing autophagy, mitophagy, collagen II expression and reducing the OA-related proteins, which might make PHF23 a possible therapeutic target for OA [142]. Fan et al. [143] demonstrated that $17 \beta$-estradiol, a steroid hormone, inhibited mitophagy by activating the PI3K/AKT/ mTOR signaling pathway via the G-protein coupled estrogen receptor to exert protective effect on chondrocytes in OA. In addition, Blanco et al. [144] indicated their hypothetic view on the key role of AMPK-SIRT-Parkin in regulating mitochondrial function and defensing against excessive ROS in the chondrocytes of OA (Figure 6A).

\section{RA}

RA is a chronic inflammatory condition [10, 145], during which osteoclasts differentiation is induced, and bone resorption is activated, mediated by the secretion of pro-inflammatory factors and RANKL in fibroblast-like synoviocytes. Evidence has suggested that autophagy is associated with the survival of fibroblast-like synoviocytes in RA [146]. Autophagy also plays a key role in the maintenance of the central tolerance mechanism. The inhibition of autophagy blocks osteoclast differentiation from mouse macrophages and reduces bone erosion and the number of osteoclasts in a RA mouse model [147].

Similar to OA, autophagy is also involved in the mediation of the secretion and release of inflammatory cytokines in RA. In circulating immune 
cells, especially the $\mathrm{CD}^{+} \mathrm{T}$ and $\mathrm{CD}^{+} \mathrm{T}$ cells [148], increased LC3-II expression levels and decreased p62 expression levels indicated a higher level of autophagic flux in RA patients compared to healthy human [148]. Lee et al. [149] found pretreatment of RA fibroblast-like synoviocytes with brazilin induced the activation of autophagy by inhibiting TNF-induced phosphorylation and degradation of IkBa (inhibitor of NF-kB) in these synoviocytes, and it could result in a significantly decrease of the secretion of TNF-induced proinflammatory cytokines IL-6 and IL-8. Liu et al. [150] found in the mice with collageninduced arthritis, polypeptide composite selenium nanoparticles induced vascular endothelial cells to produce NO (nitric oxide), NO induced AMPK-a phosphorylation, which could inhibit mTOR phosphorylation, increase autophagy flux, inhibit NF-kB-p65 phosphorylation and reduce the level of inflammatory cytokines (IL-1 $\beta$, IL-6 and TNF- $\alpha$ ) in the polypeptide composite selenium nanoparticles treated mice RA immune cells. Zhou et al. [151] similarly found fumagillin prodrug nano-therapy induced endothelial NO production and activated AMPK, which subsequently modulated macrophage inflammatory response by inhibiting mTOR activity, increased autophagic flux, decreased degradation of ІкB kinase, then suppressed the NF-кB-p65 signaling pathway and inflammatory cytokines release. Melatonin has beneficial effects on macrophage-

\section{(A). AMPK/SIRT/Parkin}

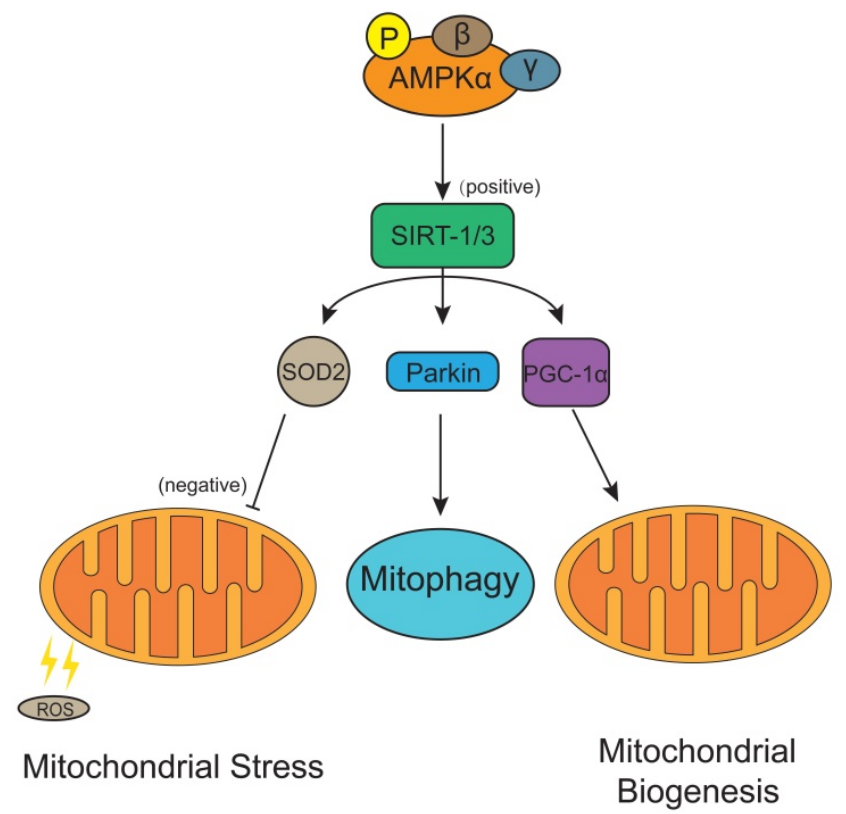

associated diseases by regulating macrophage responses [152]. Mitochondrial dynamics and mitophagy are shown to be involved in the regulatory functions of melatonin on macrophage polarization [152]. Whether melatonin can regulate mitophagy to treat $\mathrm{RA}$ requires more studies.

Apoptosis defect is one of the causes of synovial hyperplasia in RA. Xu et al. [153] found a significant reverse correlation between apoptosis and autophagy in RA synovial tissues, which may be due to the deregulation of microRNA-30a targeting Beclin-1. Meanwhile, Fan et al. [154] also found that hypoxia could induce autophagy and elevate the expression of peptidyl arginine deiminase type IV (PADI4), as well as promoting the proliferation of fibroblast-like synoviocytes in a RA rat model. Consistently, the knockdown of PADI4 was demonstrated to inhibit the hypoxia-induced autophagy and promote the apoptosis of fibroblast-like synoviocytes [154]. Other evidence suggests that the rate of apoptosis in RA fibroblast-like synoviocytes is inversely related to the expression of Beclin-1 and LC3. Furthermore, the inhibition of protease system activation in fibroblast synovial cells resulted in increased LC3 expression and prolonged synovial cell lifespan [155]. These studies suggested that increased autophagy in fibroblast-like synoviocytes contributes to synovial hyperplasia which promotes RA-associated synovitis.

\section{(B). PI3K/AKT/mTOR}

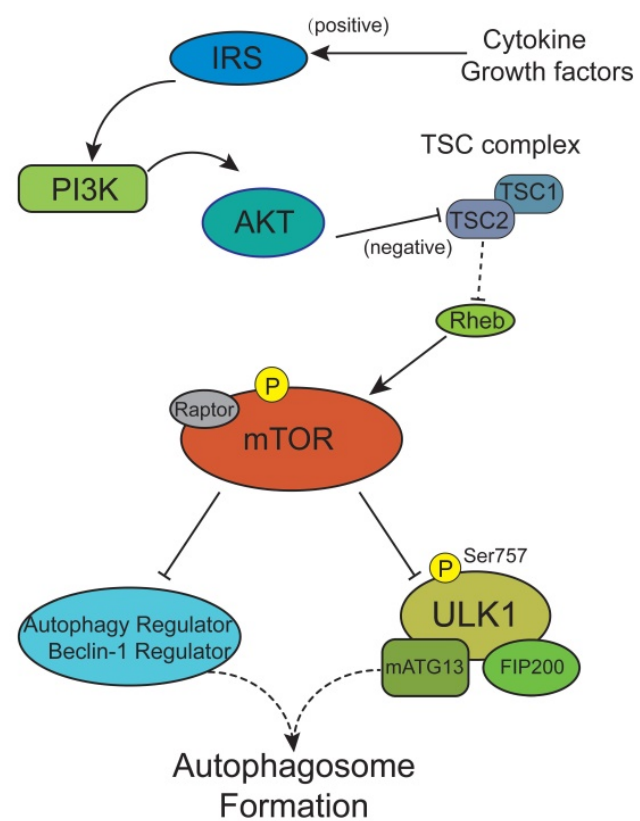

Figure 6. Autophagy regulatory signaling pathways are involved in the pathological process of osteoarthritis/rheumatoid arthritis (OA/RA). (A) The AMPK/SIRT signaling pathway in OA chondrocytes regulates the mitochondrial function, mitophagy and mitochondrial biogenesis and defenses against excessive ROS mediated by superoxide dismutase 2/Parkin/ peroxisome proliferator-activated receptor gamma coactivator-1a (SOD2/Parkin/PGC-1a). (B) The phosphatidylinositol 3-kinase/protein kinase $\mathrm{B} / \mathrm{mTOR}$ (PI3K/AKT/mTOR) signaling pathway. Cytokines or growth factors (such as insulin) activate AKT signaling via PI3K through binging to insulin receptor substrate protein (IRS). Activated AKT inhibits TSC1/2 complex activity on Rheb, stimulating the activation of mTOR, which deactivates the ULK1/mATG13/FIP200 protein kinase complex, autophagy regulator and Beclin-1 regulator to prevent autophagosome formation. 
In RA, altered autophagy levels are found not only in fibroblast-like synoviocytes but also in chondrocytes. The active participation of chondrocytes in local inflammation play a key role in RA, may cause a disruption of cartilage repair mechanisms resulting in cartilage destruction [156]. Feng et al. found artesunate decreased $\mathrm{PI} 3 \mathrm{~K} / \mathrm{AKT} / \mathrm{mTOR}$ signals (Figure 6B) and increased B-cell lymphoma/leukemia-2-associated $X$ protein (Bax), LC3-II/LC3-I and Beclin-1 in chondrocytes, resulting in inhibited chondrocyte proliferation and accelerated cell apoptosis and autophagy [157]. It may indicate that treatment targeting chondrocytes in RA is a novel therapeutic strategy for the treatment of RA and the functional repair of bone inflammation.

\section{Conclusion and prospects}

The osteoblasts and osteoclasts are important cellular components of bone. Osteoblasts, derived from bone marrow mesenchymal stem cells, are the crucial source of osteocytes, which represent the terminally differentiated state of the osteoblast lineage and the most abundant type of cells in bone. However, the osteoclasts, which derived from hematopoietic stem cells, are the only type of cell responsible for bone resorption. The balance between bone formation by osteoblasts and bone resorption by osteoclasts constitute the two major processes of bone remodeling. The level of autophagy and mitophagy could significantly affect the function and viability of osteoblasts and osteoclasts. Multiple factors are involved in regulating autophagy in cells, such as widely researched wear particles [113], hypoxic conditions [111], starvation conditions [105], even microgravity [158]. Evidence suggested that wear particle induced osteoblasts apoptosis [19] and osteoclasts formation and differentiation through enhanced autophagy [89]. These studies demonstrated that the inhibition of autophagy or mitophagy, by an autophagy inhibitor 3-methyladenine or siRNA knockdown of Atg5, could dramatically reduce the role of autophagy on function and proliferation of osteoblasts and osteoclasts in vitro and the severity of osteolysis in vivo. In contrast, though the role of mitophagy on bone cells is less studied, previous research has indicated that mitochondrial function and quantity are important to maintain osteoblasts and osteoclasts; therefore, more investigation would be desired to illustrate the involvement of mitophagy on bone metabolic disorders in the future. Up to date, no effective drug therapy to prevent or inhibit bone metabolic disorders has been confirmed. Surgical revision remains as the only treatment for terminal and severe bone metabolic disorders, such as aseptic loosening following THA.
To date, in multitudinous signaling pathways involved in the autophagy and mitophagy, PINK1/ Parkin and SIRT1 signaling pathways are the most extensively studied regulation factors of mitophagy, because of their crucial role in regulating mitophagy. In addition, other autophagy regulated factors, such as p62/SQSTM1, Beclin1, AMPK, mTOR1 and ATGs, form a complex and tightly connected regulatory network to mediate autophagy $[157,159]$. In this review, we summarize and discuss some signaling pathways that have been proved to have regulatory effects on autophagy and mitophagy in osteoblasts and osteoclasts during the physiopathologic change of bone metabolic disorders. Together, this evidence suggests a vital role of autophagy and mitophagy in bone metabolic disorders. Our previous study found that wear particles induced abnormal autophagy in osteoblasts and osteoclasts which could disrupt homeostasis of bone metabolism and lead to osteolysis, but the underlying mechanism remains unclear. Moreover, given the critical role of inflammatory cytokine secretion mediated by autophagy, inflammation could constitute a key factor in aseptic loosening pathology. This requires further study on the role of autophagy and mitophagy in bone metabolic disorders induced by stimulating factor like wear particles, to find specific regulated factor as a new underlying therapeutic target for preventing and controlling pathological process of osteolysis following THA.

\section{Abbreviations}

AMPK: AMP-activated protein kinase; Atg: autophagy related genes; ATP: adenosine triphosphate; Beclin-1: Bcl-2 interacting coiled-coil protein; ETC: electron transport chain; FOXO: Forkhead box O; HIF-1a: hypoxia-inducible factor-1a; HT: hydroxytyrosol; IL: interleukin; IMM: inner mitochondrial membrane; LC3: light chain 3; MAPK: mitogen-activated protein kinase; MSCs: mesenchymal stem cells; mTOR: mammalian target of rapamycin; MTS: mitochondrial targeting sequence; NAD: nicotinamide adenine dinucleotide; OA: osteoarthritis; OMM: outer mitochondrial membrane; OPG: osteoprotegerin; SQSTM1: sequestosome 1; p70S6K: 70-kDa ribosomal protein S6 kinase; Parkin: Parkinson disease (autosomal recessive, juvenile) 2; PINK1: PTEN-induced putative kinase 1; RA: rheumatic arthritis; RANKL: receptor activator of nuclear factor-KB ligand; Rheb: Ras homolog enriched in brain; RING: really interesting new gene; ROS: reactive oxygen species; Ser: serine; SIRT1: Sirtuin 1; SOD2: superoxide dismutase 2; THA: total hip arthroplasty; TM: transmembrane; TNF-a: tumor necrosis factor- $\alpha$; TOM: translocase of the outer 
membrane; TSC: tuberous sclerosis complex; UBL: ubiquitin-like; ULK1: UNC-51 like autophagy activating kinase $1 ; \Delta \Psi \mathrm{m}$ : mitochondrial membrane potential.

\section{Acknowledgements}

This work was supported by the National Natural Science Foundation of China Youth Science Foundation (grant number 81802222), the National Natural Science Foundation of China (81702191), the Natural Science Foundation of Guangdong Province (2018A030310694, 2020A1515010268, 2020A1515011031), Provincial Science and Technology Major Project of Guangdong (2015B020225007), the Foundation of Traditional Chinese Medicine of Guangdong Province (20191004), the Scientific Foundation of Guangdong Provincial People's Hospital (2017bp01), the Outstanding Young Talents Foundation of Guangdong Provincial People's Hospital (KJ012019091), the Program of Science and Technology of Guangzhou (201904010424), the Guangdong Medical Science and Technology Research Foundation (2018114214430383) and the Fundamental Research Funds for the Central Universities, South China University of Technology (2018MS70).

\section{Competing Interests}

The authors have declared that no competing interest exists.

\section{References}

1. Miyazaki T, Iwasawa M, Nakashima T, Mori S, Shigemoto K, Nakamura H, et al. Intracellular and extracellular ATP coordinately regulate the inverse correlation between osteoclast survival and bone resorption. J Biol Chem. 2012; 287: 37808-23.

2. Vasilyeva LV, Lakhin DI. Clinical features of osteoarthritis in patients with metabolic syndrome. Ter Arkh. 2017; 89: 65-8.

3. Sacitharan PK. Ageing and Osteoarthritis. Subcell Biochem. 2019; 91: 123-59.

4. Martel-Pelletier J, Barr AJ, Cicuttini FM, Conaghan PG, Cooper C, Goldring MB, et al. Osteoarthritis. Nat Rev Dis Primers. 2016; 2: 16072.

5. Pope RM. Apoptosis as a therapeutic tool in rheumatoid arthritis. Nat Rev Immunol. 2002; 2: 527-35

6. Varnum C. Outcomes of different bearings in total hip arthroplasty - implant survival, revision causes, and patient-reported outcome. Dan Med J. 2017; 64.

7. Veronesi F, Tschon M, Fini M. Gene Expression in Osteolysis: Review on the Identification of Altered Molecular Pathways in Preclinical and Clinical Studies. Int J Mol Sci. 2017; 18.

8. Purdue PE, Koulouvaris P, Potter HG, Nestor BJ, Sculco TP. The cellular and molecular biology of periprosthetic osteolysis. Clin Orthop Relat Res. 2007; 454: 251-61.

9. Firestein GS. Evolving concepts of rheumatoid arthritis. Nature. 2003; 423: 356-61.

10. Bottini N, Firestein GS. Duality of fibroblast-like synoviocytes in RA: passive responders and imprinted aggressors. Nat Rev Rheumatol. 2013; 9: 24-33.

11. Glick D, Barth S, Macleod KF. Autophagy: cellular and molecular mechanisms. J Pathol. 2010; 221: 3-12.

12. Mizushima N, Komatsu M. Autophagy: renovation of cells and tissues. Cell. 2011; 147: 728-41.

13. Parzych KR, Klionsky DJ. An overview of autophagy: morphology, mechanism, and regulation. Antioxid Redox Signal. 2014; 20: 460-73.

14. Boya P, Reggiori F, Codogno P. Emerging regulation and functions of autophagy. Nat Cell Biol. 2013; 15: 713-20.

15. Wang Y, Liu N, Lu B. Mechanisms and roles of mitophagy in neurodegenerative diseases. CNS Neurosci Ther. 2019

16. Esteban-Martinez L, Sierra-Filardi E, McGreal RS, Salazar-Roa M, Marino G, Seco E, et al. Programmed mitophagy is essential for the glycolytic switch during cell differentiation. Embo Journal. 2017; 36: 1688-706.
17. Heynen MJ, Tricot G, Verwilghen RL. Autophagy of mitochondria in rat bone marrow erythroid cells. Relation to nuclear extrusion. Cell Tissue Res. 1985; 239: 235-9.

18. Pua HH, Guo J, Komatsu M, He YW. Autophagy is essential for mitochondrial clearance in mature T lymphocytes. J Immunol. 2009; 182: 4046-55.

19. Wang Z, Liu N, Liu K, Zhou G, Gan J, Wang Z, et al. Autophagy mediated CoCrMo particle-induced peri-implant osteolysis by promoting osteoblast apoptosis. Autophagy. 2015; 11: 2358-69.

20. Wang Z, Deng Z, Gan J, Zhou G, Shi T, Wang Z, et al. TiAl6V4 particles promote osteoclast formation via autophagy-mediated downregulation of interferon-beta in osteocytes. Acta Biomater. 2017; 48: 489-98.

21. Zaidi M. Skeletal remodeling in health and disease. Nat Med. 2007; 13: 791-801.

22. Tanaka Y, Nakayamada S, Okada Y. Osteoblasts and osteoclasts in bone remodeling and inflammation. Curr Drug Targets Inflamm Allergy. 2005; 4: $325-8$.

23. Deng Z, Wang Z, Jin J, Wang Y, Bao N, Gao Q, et al. SIRT1 protects osteoblasts against particle-induced inflammatory responses and apoptosis in aseptic prosthesis loosening. Acta Biomater. 2017; 49: 541-54.

24. Liu N, Meng J, Wang Z, Zhou G, Shi T, Zhao J. Autophagy mediated $\mathrm{TiAl}(6) \mathrm{V}(4)$ particle-induced peri-implant osteolysis by promoting expression of TNF-alpha. Biochem Biophys Res Commun. 2016; 473: 133-9.

25. Negishi-Koga T, Shinohara M, Komatsu N, Bito H, Kodama T, Friedel RH, et al. Suppression of bone formation by osteoclastic expression of semaphorin 4D. Nat Med. 2011; 17: 1473-80

26. Deter RL, De Duve C. Influence of glucagon, an inducer of cellular autophagy, on some physical properties of rat liver lysosomes. J Cell Biol. 1967; 33: 437-49.

27. Nakatogawa H, Suzuki K, Kamada Y, Ohsumi Y. Dynamics and diversity in autophagy mechanisms: lessons from yeast. Nat Rev Mol Cell Biol. 2009; 10: 458-67.

28. Yu L, Chen Y, Tooze SA. Autophagy pathway: Cellular and molecular mechanisms. Autophagy. 2018; 14: 207-15.

29. Wang Y, Qin ZH. Coordination of autophagy with other cellular activities. Acta Pharmacol Sin. 2013; 34: 585-94.

30. Lemasters JJ. Selective mitochondrial autophagy, or mitophagy, as a targeted defense against oxidative stress, mitochondrial dysfunction, and aging. Rejuvenation Res. 2005; 8: 3-5.

31. Hom J, Sheu SS. Morphological dynamics of mitochondria--a special emphasis on cardiac muscle cells. J Mol Cell Cardiol. 2009; 46: 811-20.

32. Martinez-Reyes I, Cuezva JM. The H(+)-ATP synthase: a gate to ROS-mediated cell death or cell survival. Biochim Biophys Acta. 2014; 1837: 1099-112.

33. Zhao RZ, Jiang S, Zhang L, Yu ZB. Mitochondrial electron transport chain, ROS generation and uncoupling (Review). Int J Mol Med. 2019; 44: 3-15.

34. Pyatrikas DV, Fedoseeva IV, Varakina NN, Rusaleva TM, Stepanov AV, Fedyaeva AV, et al. Relation between cell death progression, reactive oxygen species production and mitochondrial membrane potential in fermenting Saccharomyces cerevisiae cells under heat-shock conditions. Fems Microbiol Lett. 2015; 362.

35. Korshunov SS, Skulachev VP, Starkov AA. High protonic potential actuates a mechanism of production of reactive oxygen species in mitochondria. FEBS Lett. 1997; 416: 15-8.

36. Suski JM, Lebiedzinska M, Bonora M, Pinton P, Duszynski J, Wieckowski MR. Relation between mitochondrial membrane potential and ROS formation. Methods Mol Biol. 2012; 810: 183-205.

37. Porter GA, Jr., Hom J, Hoffman D, Quintanilla R, de Mesy Bentley K, Sheu SS. Bioenergetics, mitochondria, and cardiac myocyte differentiation. Prog Pediatr Cardiol. 2011; 31: 75-81.

38. Guo Y, Yang TL, Liu YZ, Shen H, Lei SF, Yu N, et al. Mitochondria-wide association study of common variants in osteoporosis. Ann Hum Genet. 2011; 75: 569-74.

39. Hui W, Young DA, Rowan AD, Xu X, Cawston TE, Proctor CJ. Oxidative changes and signalling pathways are pivotal in initiating age-related changes in articular cartilage. Ann Rheum Dis. 2016; 75: 449-58.

40. Collins JA, Diekman BO, Loeser RF. Targeting aging for disease modification in osteoarthritis. Curr Opin Rheumatol. 2018; 30: 101-7.

41. Goldman SJ, Taylor R, Zhang Y, Jin S. Autophagy and the degradation of mitochondria. Mitochondrion. 2010; 10: 309-15.

42. Shen $\mathrm{Z}$, Zheng $\mathrm{Y}, \mathrm{Wu} \mathrm{J}$, Chen $\mathrm{Y}, \mathrm{Wu} \mathrm{X}$, Zhou Y, et al. PARK2-dependent mitophagy induced by acidic postconditioning protects against focal cerebral ischemia and extends the reperfusion window. Autophagy. 2017; 13: 473-85.

43. Wai T, Garcia-Prieto J, Baker MJ, Merkwirth C, Benit P, Rustin P, et al Imbalanced OPA1 processing and mitochondrial fragmentation cause heart failure in mice. Science. 2015; 350: aad0116.

44. Gao J, Feng Z, Wang X, Zeng M, Liu J, Han S, et al. SIRT3/SOD2 maintains osteoblast differentiation and bone formation by regulating mitochondrial stress. Cell Death Differ. 2018; 25: 229-40.

45. Ploumi C, Daskalaki I, Tavernarakis N. Mitochondrial biogenesis and clearance: a balancing act. FEBS J. 2017; 284: 183-95.

46. Liu X, Ye B, Miller S, Yuan H, Zhang H, Tian L, et al. Ablation of ALCAT1 mitigates hypertrophic cardiomyopathy through effects on oxidative stress and mitophagy. Mol Cell Biol. 2012; 32: 4493-504.

47. Sun X, Yang X, Zhao Y, Li Y, Guo L. Effects of $17 \beta$-Estradiol on Mitophagy in the Murine MC3T3-E1 Osteoblast Cell Line is Mediated via G Protein-Coupled 
Estrogen Receptor and the ERK1/2 Signaling Pathway. Med Sci Monit. 2018; 24: 903-11.

48. Zhao W, Zhang W, Ma H, Yang M. NIPA2 regulates osteoblast function by modulating mitophagy in type 2 diabetes osteoporosis. Sci Rep. 2020; 10: 3078.

49. Yang X, Jiang T, Wang Y, Guo L. The Role and Mechanism of SIRT1 in Resveratrol-regulated Osteoblast Autophagy in Osteoporosis Rats. Sci Rep. 2019; 9: 18424.

50. Yang CN, Kok SH, Wang HW, Chang JZ, Lai EH, Shun CT, et al. Simvastatin alleviates bone resorption in apical periodontitis possibly by inhibition of mitophagy-related osteoblast apoptosis. Int Endod J. 2019; 52: 676-88.

51. Piemontese M, Onal M, Xiong J, Han L, Thostenson JD, Almeida M, et al. Low bone mass and changes in the osteocyte network in mice lacking autophagy in the osteoblast lineage. Sci Rep. 2016; 6: 24262.

52. Bartolome A, Lopez-Herradon A, Portal-Nunez S, Garcia-Aguilar A, Esbrit P, Benito $\mathrm{M}$, et al. Autophagy impairment aggravates the inhibitory effects of high glucose on osteoblast viability and function. Biochem J. 2013; 455: 329-37.

53. Narendra DP, Jin SM, Tanaka A, Suen DF, Gautier CA, Shen J, et al. PINK1 Is Selectively Stabilized on Impaired Mitochondria to Activate Parkin. Plos Biol. 2010; 8 .

54. Kawajiri S, Saiki S, Sato S, Sato F, Hatano T, Eguchi H, et al. PINK1 is recruited to mitochondria with parkin and associates with LC3 in mitophagy. Febs Letters. 2010; 584: 1073-9.

55. Narendra D, Tanaka A, Suen DF, Youle RJ. Parkin is recruited selectively to impaired mitochondria and promotes their autophagy. Journal of Cell Biology. 2008; 183: 795-803.

56. Bras J, Guerreiro R, Hardy J. SnapShot: Genetics of Parkinson's disease. Cell. 2015; 160: 570- e1.

57. Eiyama A, Okamoto K. PINK1/Parkin-mediated mitophagy in mammalian cells. Curr Opin Cell Biol. 2015; 33: 95-101.

58. Greene AW, Grenier K, Aguileta MA, Muise S, Farazifard R, Haque ME, et al. Mitochondrial processing peptidase regulates PINK1 processing, import and Parkin recruitment. EMBO Rep. 2012; 13: 378-85.

59. Yamano K, Youle RJ. PINK1 is degraded through the N-end rule pathway. Autophagy. 2013; 9: 1758-69.

60. Chaugule VK, Burchell L, Barber KR, Sidhu A, Leslie SJ, Shaw GS, et al. Autoregulation of Parkin activity through its ubiquitin-like domain. EMBO J. 2011; 30: 2853-67.

61. Lazarou M, Jin SM, Kane LA, Youle RJ. Role of PINK1 binding to the TOM complex and alternate intracellular membranes in recruitment and activation of the E3 ligase Parkin. Dev Cell. 2012; 22: 320-33.

62. Okatsu K, Uno M, Koyano F, Go E, Kimura M, Oka T, et al. A dimeric PINK1-containing complex on depolarized mitochondria stimulates Parkin recruitment. J Biol Chem. 2013; 288: 36372-84.

63. Kondapalli C, Kazlauskaite A, Zhang N, Woodroof HI, Campbell DG, Gourlay $\mathrm{R}$, et al. PINK1 is activated by mitochondrial membrane potential depolarization and stimulates Parkin E3 ligase activity by phosphorylating Serine 65. Open Biol. 2012; 2.

64. Geisler S, Holmstrom KM, Skujat D, Fiesel FC, Rothfuss OC, Kahle PJ, et al. PINK1/Parkin-mediated mitophagy is dependent on VDAC1 and p62/SQSTM1. Nat Cell Biol. 2010; 12: 119-31.

65. Gegg ME, Cooper JM, Chau KY, Rojo M, Schapira AH, Taanman JW. Mitofusin 1 and mitofusin 2 are ubiquitinated in a PINK1/parkin-dependent manner upon induction of mitophagy. Hum Mol Genet. 2010; 19: 4861-70.

66. Sarraf SA, Raman M, Guarani-Pereira V, Sowa ME, Huttlin EL, Gygi SP, et al. Landscape of the PARKIN-dependent ubiquitylome in response to mitochondrial depolarization. Nature. 2013; 496: 372-6.

67. Nixon RA. The role of autophagy in neurodegenerative disease. Nat Med. 2013; 19: 983-97.

68. Ivankovic D, Chau KY, Schapira AH, Gegg ME. Mitochondrial and lysosomal biogenesis are activated following PINK1/parkin-mediated mitophagy. J Neurochem. 2016; 136: 388-402.

69. Ding WX, Ni HM, Li M, Liao Y, Chen X, Stolz DB, et al. Nix is critical to two distinct phases of mitophagy, reactive oxygen species-mediated autophagy induction and Parkin-ubiquitin-p62-mediated mitochondrial priming. J Biol Chem. 2010; 285: 27879-90.

70. Kazlauskaite A, Martinez-Torres RJ, Wilkie S, Kumar A, Peltier J, Gonzalez A, et al. Binding to serine 65-phosphorylated ubiquitin primes Parkin for optimal PINK1-dependent phosphorylation and activation. EMBO Rep. 2015; 16: 939-54.

71. F K, K O, H K, Y T, E G, M K, et al. Ubiquitin is phosphorylated by PINK1 to activate parkin. Nature. 2014; 510: 162-6.

72. LA K, M L, AI F, Y L, K Y, SA S, et al. PINK1 phosphorylates ubiquitin to activate Parkin E3 ubiquitin ligase activity. The Journal of cell biology. 2014; 205: $143-53$

73. A K, C K, R G, DG C, MS R, K H, et al. Parkin is activated by PINK1-dependent phosphorylation of ubiquitin at Ser65. The Biochemical journal. 2014; 460: 127-39.

74. Shi RY, Zhu SH, Li V, Gibson SB, Xu XS, Kong JM. BNIP3 interacting with LC3 triggers excessive mitophagy in delayed neuronal death in stroke. CNS Neurosci Ther. 2014; 20: 1045-55.

75. Ryan BJ, Hoek S, Fon EA, Wade-Martins R. Mitochondrial dysfunction and mitophagy in Parkinson's: from familial to sporadic disease. Trends Biochem Sci. 2015 ; 40: 200-10.
76. Koentioro B, Park JS, Sue CM. Nix restores mitophagy and mitochondrial function to protect against PINK1/Parkin-related Parkinson's disease. Sci Rep. 2017; 7: 44373 .

77. Marycz K, Kornicka K, Maredziak M, Golonka P, Nicpon J. Equine metabolic syndrome impairs adipose stem cells osteogenic differentiation by predominance of autophagy over selective mitophagy. J Cell Mol Med. 2016; 20: $2384-404$.

78. Finkel T, Deng CX, Mostoslavsky R. Recent progress in the biology and physiology of sirtuins. Nature. 2009; 460: 587-91.

79. Rine J, Strathern JN, Hicks JB, Herskowitz I. A suppressor of mating-type locus mutations in Saccharomyces cerevisiae: evidence for and identification of cryptic mating-type loci. Genetics. 1979; 93: 877-901.

80. Cohen-Kfir E, Artsi H, Levin A, Abramowitz E, Bajayo A, Gurt I, et al. Sirt1 Is a Regulator of Bone Mass and a Repressor of Sost Encoding for Sclerostin, a Bone Formation Inhibitor. Endocrinology. 2011; 152: 4514-24.

81. Sun W, Qiao W, Zhou B, Hu Z, Yan Q, Wu J, et al. Overexpression of Sirt1 in mesenchymal stem cells protects against bone loss in mice by FOXO3a deacetylation and oxidative stress inhibition. Metabolism. 2018; 88: 61-71.

82. Tang BL. Sirt1 and the Mitochondria. Mol Cells. 2016; 39: 87-95.

83. Yoshii SR, Mizushima N. Autophagy machinery in the context of mammalian mitophagy. Biochim Biophys Acta. 2015; 1853: 2797-801.

84. Jang SY, Kang HT, Hwang ES. Nicotinamide-induced mitophagy: event mediated by high NAD+/NADH ratio and SIRT1 protein activation. J Biol Chem. 2012; 287: 19304-14.

85. Das S, Mitrovsky G, Vasanthi HR, Das DK. Antiaging properties of a grape-derived antioxidant are regulated by mitochondrial balance of fusion and fission leading to mitophagy triggered by a signaling network of Sirt1-Sirt3-Foxo3-PINK1-PARKIN. Oxid Med Cell Longev. 2014; 2014: 345105.

86. Di Sante G, Pestell TG, Casimiro MC, Bisetto S, Powell MJ, Lisanti MP, et al. Loss of Sirt1 promotes prostatic intraepithelial neoplasia, reduces mitophagy, and delays PARK2 translocation to mitochondria. Am J Pathol. 2015; 185: 266-79.

87. Gomez-Puerto MC, Verhagen LP, Braat AK, Lam EW, Coffer PJ, Lorenowicz MJ. Activation of autophagy by FOXO3 regulates redox homeostasis during osteogenic differentiation. Autophagy. 2016; 12: 1804-16.

88. Wei M, Duan D, Liu Y, Wang Z, Li Z. Autophagy may protect MC3T3-E1 cells from fluoride-induced apoptosis. Molecular medicine reports. 2014; 9: 2309-15.

89. Su B, Li D, Xu J, Zhang Y, Cai Z, Kauther MD, et al. Wear particles enhance autophagy through up-regulation of CD147 to promote osteoclastogenesis. Iran J Basic Med Sci. 2018; 21: 806-12.

90. Zhao SJ, Kong FQ, Cai W, Xu T, Zhou ZM, Wang ZB, et al. GIT1 contributes to autophagy in osteoclast through disruption of the binding of Beclin1 and Bcl2 under starvation condition. Cell Death Dis. 2018; 9: 1195.

91. Xue Y, Liang Z, Fu X, Wang T, Xie Q, Ke D. IL-17A modulates osteoclast precursors' apoptosis through autophagy-TRAF3 signaling during osteoclastogenesis. Biochem Biophys Res Commun. 2019; 508: 1088-92.

92. Jha P, Das H. KLF2 in Regulation of NF-kappaB-Mediated Immune Cell Function and Inflammation. Int J Mol Sci. 2017; 18.

93. Laha D, Deb M, Das H. KLF2 (kruppel like factor 2 [lung]) regulates osteoclastogenesis by modulating autophagy. Autophagy. 2019; 12: 1-13.

94. Arai A, Kim S, Goldshteyn V, Kim T, Park NH, Wang CY, et al. Beclin1 modulates bone homeostasis by regulating osteoclast and chondrocyte differentiation. J Bone Miner Res. 2019.

95. Li RF, Chen G, Ren JG, Zhang W, Wu ZX, Liu B, et al. The adaptor protein p62 is involved in RANKL-induced autophagy and osteoclastogenesis. J Histochem Cytochem. 2014; 62: 879-88

96. Kim CJ, Shin SH, Kim BJ, Kim CH, Kim JH, Kang HM, et al. The Effects of Kaempferol-Inhibited Autophagy on Osteoclast Formation. Int J Mol Sci. 2018; 19(1)125.

97. Ralston SH, Layfield R. Pathogenesis of Paget disease of bone. Calcif Tissue Int. 2012; 91: 97-113.

98. Rea SL, Walsh JP, Layfield R, Ratajczak T, Xu J. New insights into the role of sequestosome 1/p62 mutant proteins in the pathogenesis of Paget's disease of bone. Endocr Rev. 2013; 34: 501-24.

99. Kobayashi $\mathrm{Y}$, Udagawa N, Takahashi N. Action of RANKL and OPG for osteoclastogenesis. Crit Rev Eukaryot Gene Expr. 2009; 19: 61-72.

100. Fu YX, Gu JH, Zhang YR, Tong XS, Zhao HY, Yuan Y, et al. Inhibitory effects of osteoprotegerin on osteoclast formation and function under serum-free conditions. J Vet Sci. 2013; 14: 405-12.

101. Zhao H, Liu X, Zou H, Dai N, Yao L, Zhang X, et al. Osteoprotegerin disrupts peripheral adhesive structures of osteoclasts by modulating Pyk2 and Src activities. Cell Adh Migr. 2016; 10: 299-309.

102. Tong X, Zhang C, Wang D, Song R, Ma Y, Cao Y, et al. Suppression of AMP-activated protein kinase reverses osteoprotegerin-induced inhibition of osteoclast differentiation by reducing autophagy. Cell Prolif. 2020; 53: e12714.

103. Tong X, Gu J, Song R, Wang D, Sun Z, Sui C, et al. Osteoprotegerin inhibit osteoclast differentiation and bone resorption by enhancing autophagy via $\mathrm{AMPK} / \mathrm{mTOR} / \mathrm{p} 70 \mathrm{~S} 6 \mathrm{~K}$ signaling pathway in vitro. J Cell Biochem. 2018.

104. Lacher MD, Pincheira RI, Castro AF. Consequences of interrupted Rheb-to-AMPK feedback signaling in tuberous sclerosis complex and cancer. Small GTPases. 2011; 2: 211-6.

105. Cai ZY, Yang B, Shi YX, Zhang WL, Liu F, Zhao W, et al. High glucose downregulates the effects of autophagy on osteoclastogenesis via the AMPK/mTOR/ULK1 pathway. Biochem Biophys Res Commun. 2018; 503: 428-35. 
106. Kim J, Kundu M, Viollet B, Guan KL. AMPK and mTOR regulate autophagy through direct phosphorylation of Ulk1. Nat Cell Biol. 2011; 13: 132-41.

107. Ha J, Guan KL, Kim J. AMPK and autophagy in glucose/glycogen metabolism. Mol Aspects Med. 2015; 46: 46-62.

108. Kim J, Guan KL. Regulation of the autophagy initiating kinase ULK1 by nutrients: roles of mTORC1 and AMPK. Cell Cycle. 2011; 10: 1337-8.

109. Egan DF, Shackelford DB, Mihaylova MM, Gelino S, Kohnz RA, Mair W, et al. Phosphorylation of ULK1 (hATG1) by AMP-activated protein kinase connects energy sensing to mitophagy. Science. 2011; 331: 456-61.

110. Egan D, Kim J, Shaw RJ, Guan KL. The autophagy initiating kinase ULK1 is regulated via opposing phosphorylation by AMPK and mTOR. Autophagy. 2011; 7: 643-4

111. Zhao Y, Chen G, Zhang W, Xu N, Zhu JY, Jia J, et al. Autophagy regulates hypoxia-induced osteoclastogenesis through the HIF-1alpha/BNIP3 signaling pathway. J Cell Physiol. 2012; 227: 639-48.

112. Sun KT, Chen MY, Tu MG, Wang IK, Chang SS, Li CY. MicroRNA-20a regulates autophagy related protein-ATG16L1 in hypoxia-induced osteoclast differentiation. Bone. 2015; 73: 145-53.

113. Drees P, Eckardt A, Gay RE, Gay S, Huber LC. Mechanisms of disease: Molecular insights into aseptic loosening of orthopedic implants. Nat Clin Pract Rheumatol. 2007; 3: 165-71.

114. Camuzard O, Breuil V, Carle GF, Pierrefite-Carle V. Autophagy Involvement in Aseptic Loosening of Arthroplasty Components. J Bone Joint Surg Am. 2019; 101: 466-72.

115. Li, Wang C, Li Z, Wang H, He J, Zhu J, et al. Nano-sized Al2O3 particle-induced autophagy reduces osteolysis in aseptic loosening of total hip arthroplasty by negative feedback regulation of RANKL expression in fibroblasts. Cell Death Dis. 2018; 9: 840.

116. Sun X, Yang X, Zhao Y, Li Y, Guo L. Effects of 17beta-Estradiol on Mitophagy in the Murine MC3T3-E1 Osteoblast Cell Line is Mediated via G Protein-Coupled Estrogen Receptor and the ERK1/2 Signaling Pathway. Med Sci Monit. 2018; 24: 903-11.

117. Shen G, Ren H, Qiu T, Liang D, Xie B, Zhang Z, et al. Implications of the Interaction Between miRNAs and Autophagy in Osteoporosis. Calcif Tissue Int. 2016; 99: 1-12

118. Qi M, Zhang L, Ma Y, Shuai Y, Li L, Luo K, et al. Autophagy Maintains the Function of Bone Marrow Mesenchymal Stem Cells to Prevent Estrogen Deficiency-Induced Osteoporosis. Theranostics. 2017; 7: 4498-516.

119. Yang YH, Li B, Zheng XF, Chen JW, Chen K, Jiang SD, et al. Oxidative damage to osteoblasts can be alleviated by early autophagy through the endoplasmic reticulum stress pathway--implications for the treatment of osteoporosis. Free Radic Biol Med. 2014; 77: 10-20.

120. Zainabadi K. Drugs targeting SIRT1, a new generation of therapeutics for osteoporosis and other bone related disorders? Pharmacol Res. 2019; 143: 97-105.

121. Chen W, Sun Y, Gu X, Hao Y, Liu X, Lin J, et al. Conditioned medium of mesenchymal stem cells delays osteoarthritis progression in a rat model by protecting subchondral bone, maintaining matrix homeostasis, and enhancing autophagy. J Tissue Eng Regen Med. 2019.

122. Chen Z, Li C, Qian YH, Fu Y, Feng ZM. Enhancement of autophagy flux by isopsoralen ameliorates interleukin-1beta-stimulated apoptosis in rat chondrocytes. J Asian Nat Prod Res. 2020; 22(2): 1-14.

123. Hu J, Cui W, Ding W, Gu Y, Wang Z, Fan W. Globular Adiponectin Attenuated H2O2-Induced Apoptosis in Rat Chondrocytes by Inducing Autophagy Through the AMPK/ mTOR Pathway. Cell Physiol Biochem. 2017; 43: 367-82

124. Luo C, Li Y, Wang H, Cui Y, Feng Z, Li H, et al. Hydroxytyrosol promotes superoxide production and defects in autophagy leading to anti-proliferation and apoptosis on human prostate cancer cells. Curr Cancer Drug Targets. 2013; 13: 625-39.

125. Zhi LQ, Yao SX, Liu HL, Li M, Duan N, Ma JB. Hydroxytyrosol inhibits the inflammatory response of osteoarthritis chondrocytes via SIRT6-mediated autophagy. Molecular medicine reports. 2018; 17: 4035-42.

126. Cetrullo S, D'Adamo S, Guidotti S, Borzi RM, Flamigni F. Hydroxytyrosol prevents chondrocyte death under oxidative stress by inducing autophagy through sirtuin 1-dependent and -independent mechanisms. Biochim Biophys Acta. 2016; 1860: 1181-91.

127. Settembre C, Di Malta C, Polito VA, Garcia Arencibia M, Vetrini F, Erdin S, et al. TFEB links autophagy to lysosomal biogenesis. Science. 2011; 332: 1429-33.

128. Zheng G, Zhan Y, Li X, Pan Z, Zheng F, Zhang Z, et al. TFEB, a potential therapeutic target for osteoarthritis via autophagy regulation. Cell Death Dis. 2018; 9 : 858

129. Kapoor M, Martel-Pelletier J, Lajeunesse D, Pelletier JP, Fahmi H. Role of proinflammatory cytokines in the pathophysiology of osteoarthritis. Nat Rev Rheumatol. 2011; 7: 33-42.

130. Xue JF, Shi ZM, Zou J, Li XL. Inhibition of PI3K/AKT/mTOR signaling pathway promotes autophagy of articular chondrocytes and attenuates inflammatory response in rats with osteoarthritis. Biomed Pharmacother. 2017; 89: 1252-61.

131. Khan NM, Ansari MY, Haqqi TM. Sucrose, But Not Glucose, Blocks IL1-beta-Induced Inflammatory Response in Human Chondrocytes by Inducing Autophagy via AKT/mTOR Pathway. J Cell Biochem. 2017; 118: 629-39.

132. Ansari MY, Khan NM, Haqqi TM. A standardized extract of Butea monosperma (Lam.) flowers suppresses the IL-1beta-induced expression of
IL-6 and matrix-metalloproteases by activating autophagy in human osteoarthritis chondrocytes. Biomed Pharmacother. 2017; 96: 198-207.

133. Zhao X, Li Y, Lin X, Wang J, Zhao X, Xie J, et al. Ozone induces autophagy in rat chondrocytes stimulated with IL-1beta through the AMPK/mTOR signaling pathway. Journal of pain research. 2018; 11: 3003-17.

134. Zhong G, Long H, Ma S, Shunhan Y, Li J, Yao J. miRNA-335-5p relieves chondrocyte inflammation by activating autophagy in osteoarthritis. Life sciences. 2019; 226: 164-72.

135. Mi B, Wang J, Liu Y, Liu J, Hu L, Panayi AC, et al. Icariin Activates Autophagy via Down-Regulation of the NF-kappaB Signaling-Mediated Apoptosis in Chondrocytes. Frontiers in pharmacology. 2018; 9: 605.

136. Ansari MY, Ahmad N, Haqqi TM. Butein Activates Autophagy Through AMPK/TSC2/ULK1/mTOR Pathway to Inhibit IL-6 Expression in IL-1beta Stimulated Human Chondrocytes. Cell Physiol Biochem. 2018; 49: 932-46.

137. Wu L, Liu H, Li L, Liu H, Cheng Q, Li H, et al. Mitochondrial pathology in osteoarthritic chondrocytes. Curr Drug Targets. 2014; 15: 710-9.

138. Wang C, Yang Y, Zhang Y, Liu J, Yao Z, Zhang C. Protective effects of metformin against osteoarthritis through upregulation of SIRT3-mediated PINK1/Parkin-dependent mitophagy in primary chondrocytes. Biosci Trends. 2019; 12: 605-12.

139. Huang LW, Huang TC, Hu YC, Hsieh BS, Chiu PR, Cheng HL, et al. Zinc protects chondrocytes from monosodium iodoacetate-induced damage by enhancing ATP and mitophagy. Biochem Biophys Res Commun. 2020; 521: 50-6.

140. Shin HJ, Park H, Shin N, Kwon HH, Yin Y, Hwang JA, et al. Pink1-Mediated Chondrocytic Mitophagy Contributes to Cartilage Degeneration in Osteoarthritis. J Clin Med. 2019; 8

141. Li X, Yang $\mathrm{X}$, Maimaitijuma $\mathrm{T}$, Cao $\mathrm{XY}$, Jiao $\mathrm{Y}, \mathrm{Wu} \mathrm{H}$, et al. Plant homeodomain finger protein 23 inhibits autophagy and promotes apoptosis of chondrocytes in osteoarthritis. Chin Med J (Engl). 2019; 132: 2581-7.

142. Maimaitijuma T, Yu JH, Ren YL, Yang X, Liu H, Meng ZC, et al. PHF23 negatively regulates the autophagy of chondrocytes in osteoarthritis. Life sciences. 2020; 253: 117750.

143. Fan DX, Yang XH, Li YN, Guo L. 17ß-Estradiol on the Expression of G-Protein Coupled Estrogen Receptor (GPER/GPR30) Mitophagy, and the PI3K/Akt Signaling Pathway in ATDC5 Chondrocytes In Vitro. Med Sci Monit. 2018; 24: 1936-47.

144. Blanco FJ, Rego-Perez I. Mitochondria and mitophagy: biosensors for cartilage degradation and osteoarthritis. Osteoarthritis Cartilage. 2018; 26: 989-91.

145. Karami J, Masoumi M, Khorramdelazad H, Bashiri H, Darvishi P, Sereshki $\mathrm{HA}$, et al. Role of autophagy in the pathogenesis of rheumatoid arthritis: Latest evidence and therapeutic approaches. Life sciences. 2020: 117734.

146. Wu DJ, Adamopoulos IE. Autophagy and autoimmunity. Clin Immunol. 2017; 176: 55-62.

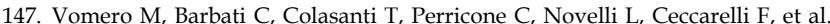
Autophagy and Rheumatoid Arthritis: Current Knowledges and Future Perspectives. Front Immunol. 2018; 9: 1577.

148. Chen YM, Chang CY, Chen HH, Hsieh CW, Tang KT, Yang MC, et al. Association between autophagy and inflammation in patients with rheumatoid arthritis receiving biologic therapy. Arthritis Res Ther. 2018; 20: 268.

149. Lee H, Kang SW, Byun HS, Jeon J, Park KA, Kang K, et al. Brazilin Limits Inflammatory Responses through Induction of Prosurvival Autophagy in Rheumatoid Fibroblast-Like Synoviocytes. PloS one. 2015; 10: e0136122.

150. Liu Y, Ma L, Zhou H, Zhu X, Yu Q, Chen X, et al. Polypeptide nano-Se targeting inflammation and theranostic rheumatoid arthritis by anti-angiogenic and NO activating AMPKalpha signaling pathway. Journal of materials chemistry B. 2018; 6: 3497-514.

151. Zhou HF, Yan H, Hu Y, Springer LE, Yang X, Wickline SA, et al. Fumagillin prodrug nanotherapy suppresses macrophage inflammatory response via endothelial nitric oxide. ACS nano. 2014; 8: 7305-17.

152. Xia Y, Chen S, Zeng S, Zhao Y, Zhu C, Deng B, et al. Melatonin in macrophage biology: Current understanding and future perspectives. Journal of pineal research. 2019; 66: e12547.

153. Xu K, Xu P, Yao JF, Zhang YG, Hou WK, Lu SM. Reduced apoptosis correlates with enhanced autophagy in synovial tissues of rheumatoid arthritis. Inflamm Res. 2013; 62: 229-37.

154. Fan T, Zhang C, Zong M, Fan L. Hypoxiainduced autophagy is inhibited by PADI4 knockdown, which promotes apoptosis of fibroblastlike synoviocytes in rheumatoid arthritis. Molecular medicine reports. 2018; 17: 5116-24.

155. Rockel JS, Kapoor M. Autophagy: controlling cell fate in rheumatic diseases. Nat Rev Rheumatol. 2016; 12: 517-31.

156. Rohner E, Matziolis G, Perka C, Fuchtmeier B, Gaber T, Burmester GR, et al. Inflammatory synovial fluid microenvironment drives primary human chondrocytes to actively take part in inflammatory joint diseases. Immunol Res. 2012; 52: 169-75.

157. Feng FB, Qiu HY. Effects of Artesunate on chondrocyte proliferation, apoptosis and autophagy through the PI3K/AKT/mTOR signaling pathway in rat models with rheumatoid arthritis. Biomed Pharmacother. 2018; 102: 1209-20.

158. Sambandam Y, Townsend MT, Pierce JJ, Lipman CM, Haque A, Bateman TA, et al. Microgravity control of autophagy modulates osteoclastogenesis. Bone. 2014; 61: 125-31.

159. Ding WX. Uncoupling AMPK from autophagy: a foe that hinders the beneficial effects of metformin treatment on metabolic syndrome-associated 
atherosclerosis? Focus on "glucose and palmitate uncouple AMPK from autophagy in human aortic endothelial cells". Am J Physiol Cell Physiol. 2015; 308: C246-8. 